NBER WORKING PAPER SERIES

\title{
A NONLINEAR CERTAINTY EQUIVALENT APPROXIMATION METHOD FOR DYNAMIC STOCHASTIC PROBLEMS
}

\author{
Yongyang Cai \\ Kenneth Judd \\ Jevgenijs Steinbuks \\ Working Paper 21590 \\ http://www.nber.org/papers/w21590 \\ NATIONAL BUREAU OF ECONOMIC RESEARCH \\ 1050 Massachusetts Avenue \\ Cambridge, MA 02138 \\ September 2015
}

We thank Thomas Hertel for his helpful comments. Cai gratefully acknowledges the National Science Foundation grant (SES-0951576). We also acknowledge the United States Department of Agriculture NIFA-AFRI grant 2015-67023-22905. This research is part of the Blue Waters sustained-petascale computing project, which is supported by the National Science Foundation (awards OCI-0725070 and ACI-1238993) and the state of Illinois. Blue Waters is a joint effort of the University of Illinois at Urbana-Champaign and its National Center for Supercomputing Applications. Responsibility for the content of the paper is the authors' alone and does not necessarily reflect the views of their institutions, or member countries of the World Bank. The views expressed herein are those of the authors and do not necessarily reflect the views of the National Bureau of Economic Research.

NBER working papers are circulated for discussion and comment purposes. They have not been peerreviewed or been subject to the review by the NBER Board of Directors that accompanies official NBER publications.

(C) 2015 by Yongyang Cai, Kenneth Judd, and Jevgenijs Steinbuks. All rights reserved. Short sections of text, not to exceed two paragraphs, may be quoted without explicit permission provided that full credit, including $(\mathbb{C}$ notice, is given to the source. 
A Nonlinear Certainty Equivalent Approximation Method for Dynamic Stochastic Problems Yongyang Cai, Kenneth Judd, and Jevgenijs Steinbuks

NBER Working Paper No. 21590

September 2015

JEL No. C61,C63,C68,E31,E52

\begin{abstract}
$\underline{\text { ABSTRACT }}$ where other algorithms, such as log-linearization, fail or are far less tractable.

Yongyang Cai

Becker Friedman Institute

University of Chicago

and

Hoover Institution

Stanford University

Stanford, CA 94305

yycai@stanford.edu

Kenneth Judd

Hoover Institution

Stanford University

Stanford, CA 94305-6010

and NBER

JUDD@HOOVER.STANFORD.EDU

Jevgenijs Steinbuks

Washington D.C.

jsteinbuks@worldbank.org
\end{abstract}

This paper introduces a nonlinear certainty equivalent approximation method for dynamic stochastic problems. We first use a novel, stable and efficient method for computing the optimal policy functions for deterministic dynamic optimization problems, and then use them as certainty-equivalent approximations for the stochastic versions. Our examples demonstrate that it can be applied to solve high-dimensional problems with up to four hundred state variables with an acceptable accuracy. This method can also be applied to solve problems with inequality constraints that occasionally bind. These features make the nonlinear certainty equivalent approximation method suitable for solving complex economic problems, 


\title{
A Nonlinear Certainty Equivalent Approximation Method for Dynamic Stochastic Problems*
}

\author{
Yongyang Cai ${ }^{\dagger} \quad$ Kenneth Judd ${ }^{\ddagger}$ Jevgenijs Steinbuks ${ }^{\S}$
}

September 23, 2015

\begin{abstract}
This paper introduces a nonlinear certainty equivalent approximation method for dynamic stochastic problems. We first use a novel, stable and efficient method for computing the optimal policy functions for deterministic dynamic optimization problems, and then use them as certainty-equivalent approximations for the stochastic versions. Our examples demonstrate that it can be applied to solve high-dimensional problems with up to four hundred state variables with an acceptable accuracy. This method can also be applied to solve problems with
\end{abstract}

*We thank Thomas Hertel for his helpful comments. Cai gratefully acknowledges the National Science Foundation grant (SES-0951576). We also acknowledge the United States Department of Agriculture NIFA-AFRI grant 2015-67023-22905. This research is part of the Blue Waters sustained-petascale computing project, which is supported by the National Science Foundation (awards OCI-0725070 and ACI-1238993) and the state of Illinois. Blue Waters is a joint effort of the University of Illinois at Urbana-Champaign and its National Center for Supercomputing Applications. Responsibility for the content of the paper is the authors' alone and does not necessarily reflect the views of their institutions, or member countries of the World Bank.

†Corresponding author: University of Chicago \& Hoover Institution. Email: yycai@stanford.edu

${ }_{\ddagger}^{\ddagger}$ Hoover Institution, Stanford University.

$\S$ Development Research Group, The World Bank. 
inequality constraints that occasionally bind. These features make the nonlinear certainty equivalent approximation method suitable for solving complex economic problems, where other algorithms, such as log-linearization, fail or are far less tractable.

Keywords: New Keynesian DSGE model, competitive equilibrium, parallel computing, sparse grid approximation, real business cycle model, occasionally binding constraints

JEL Classification: C61, C63, C68, E31, E52

\section{Introduction}

Many important problems across different fields of economics require solving dynamic stochastic general equilibrium (DSGE) or optimal decision-making problems. Numerical dynamic programming (DP) is a typical method to solve such problems, by formulating them as Bellman equation (Bellman 1957), and then solving them with value function iteration (or some accelerating methods like policy function iteration) or time iteration. ${ }^{1}$ However, implementing numerical DP faces challenging problems such as timeconsuming high-dimensional integration, keeping the shape properties of the value/policy function approximation (Cai and Judd 2013, 2015), choosing appropriate approximation domains, avoiding possible non-convergence because of accumulated approximation errors, dealing with the kinks from inequality constraints that occasionally bind, and many other challenges. Choosing a good approximation can be particularly challenging for multi-dimensional dynamic stochastic problems where the domain of state variables expands quickly over time, while a wider domain requires a higher degree approximation. Its implementation for high-dimensional problems is very timeconsuming even if we take advantage of recent innovations, such as parallel dynamic programming methods (Cai et al. 2015b) in a supercomputer or a

\footnotetext{
${ }^{1}$ For a detailed discussion of these methods, see Judd (1998), Bertsekas (2005, 2007), Rust (2008), and Cai and Judd (2014).
} 
computational grid.

Because of these challenges it is common in applied computational economics to rely on methods other than numerical DP, and sacrifice the accuracy of the results for the greater ease of numerical implementation. The most common method is log-linearization; Magill (1977) brought linearization methods for dynamic stochastic models to economics. Linear (and log-linear) approximations produce decision rules that depend only on the state of the dynamical system. They are also called certainty equivalent approximations because they do not depend on the variance of any random variable. Because of its local nature, log-linearization often fails to give a good solution on states that are not near to the steady states. ${ }^{2}$ In particular, log-linearization approximations are unsuitable for problems with inequality constraints that bind at states significantly far from the steady state.

This paper introduces a new method for solving DSGE or optimal decisionmaking problems, which we call the nonlinear certainty equivalent (NLCEQ) approximation method. Application of certainty equivalence approximations goes back to Simon (1956) and Theil (1957), who suggested solving dynamic programming problems with quadratic objectives and linear transition laws by optimizing under perfect foresight, and then using optimal deterministic forecasts for approximating unknown future values. They have also demonstrated that for some stochastic control problems, the certainty equivalent approximation is the exact solution for the optimal decision rules. ${ }^{3}$ The NLCEQ method is a natural extension of the idea of a certainty equivalent in that it solves for a nonlinear decision rule for the non-stochastic problem that is globally valid and applies this decision rule to the stochastic model. ${ }^{4}$ The

\footnotetext{
${ }^{2}$ For more detailed discussion of perturbation methods in economics, see Gaspar and Judd (1997), Jin and Judd (2002), Schmitt-Grohe and Uribe (2004), Fernandez-Villaverde and Rubio-Ramirez (2006), Kim et al. (2008), Benigno and Woodford (2012), and Den Haan and De Wind (2012).

${ }^{3}$ For a formal derivation of this result see, e.g., Hansen and Sargent (2005, section 3.2).

${ }^{4}$ Solvability of NLCEQ method follows directly from the global concavity of the value function by implicit differentiation, see e.g., Theorems 1 - 6 in Jin and Judd (2002).
} 
NLCEQ method chooses a set of states, solves the deterministic dynamic optimization problem using each of those points as the initial condition, and then use numerical approximation methods to take the results and construct a global nonlinear approximation for the value function and decision rules. This method is simple, stable, and efficient, and it can be naturally parallelized with high efficiency for high-dimensional problems. Furthermore, it does not have the challenges faced by the numerical DP. Like log-linearization (and other certainty equivalent approximations) it ignores the impact of uncertainty on the decision rule, but it is better than log-linearization over nontrivial neighborhoods of the deterministic steady state.

For deterministic dynamic problems (both social planner's problems and competitive equilibrium problems), NLCEQ can provide very accurate solution. For stochastic dynamic problems, similar to other numerical approaches, it sacrifices some accuracy of the solution for the ease of numerical implementation. However, NLCEQ has a number of important advantages over those methods.

NLCEQ is very robust to break the curse of dimensionality, and, as we show below, it can be applied to solve high-dimensional problems with up to four hundred state variables in minutes (by parallelism) with an acceptable accuracy. Moreover, NLCEQ is also appropriate for solving dynamic stochastic problems with inequality constraints that occasionally bind, where perturbation is well known for its failure to get solutions with acceptable accuracy. Furthermore, NLCEQ provides a global solution that can be used for effective impulse function analysis.

These attractive features make NLCEQ suitable for solving complex economic problems, where other algorithms fail or are too costly to get solutions with acceptable accuracy. Of course, like any numerical methods, NLCEQ has its own limitations: it may be not applicable to problems, where the presence of uncertainty in the original problem does significantly affect op- 
timal decision rules, such as dynamic portfolio optimization, ${ }^{5}$ or problems with recursive preferences (Epstein and Zin 1989). ${ }^{6}$ Like log-linearization (and other certainty equivalent approximations), NLCEQ also has a limit on its accuracy because it ignores the impact of uncertainty. However, as we show in our examples, this accuracy limit is acceptable. In fact, our results show that NLCEQ has about order-2 higher accuracy than log-linear or log-linear-quadratic perturbation methods for multi-country optimal growth problems.

In this paper we apply the NLCEQ method to solve three social planner's optimal decision-making problems and one competitive equilibrium problem. Our first example is a multi-country real business cycle (RBC) problem (Den Haan et al. 2011). We first show that NLCEQ achieves higher accuracy than log-linear or log-linear-quadratic perturbation methods in low-dimensional RBC problems, and then demonstrate that NLCEQ can solve up to a 200country RBC problem (400 state variables) in minutes by parallelism with an acceptable accuracy. Our second example is a RBC model with an occasionally binding constraint on investment (Christiano and Fisher 2000; Guerrieri and Iacoviello 2015), and it shows that NLCEQ can easily deal with occasionally binding constraints. Our third example is a dynamic stochastic model of food and clean energy (Chakravorty et al. 2008), which has inequality constraints that occasionally bind. Moreover, the problem's initial state is far away from its steady state and even its state path cannot reach its steady state in a finite time. Our results show that NLCEQ achieves an acceptable accuracy in solving these problems, which are quite challenging for all

\footnotetext{
${ }^{5}$ For example, if we apply NLCEQ to solve one portfolio problem assuming no transaction costs, its solution will be investing all money in the risky asset with the largest expected return. However, if the portfolio problem has transaction costs or does not use a HARA utility, it could also be challenging for other methods as its wealth domain will expand quickly across time and it often has occasionally binding constraints (e.g., shorting/borrowing is disallowed or limited). For more details about dynamic portfolio optimization see Infanger (2006) and Cai et al. (2013).

${ }^{6}$ Perturbation methods could get a locally accurate solution for problems with recursive preferences, see Caldara et al. (2012).
} 
other general numerical methods to the best of our knowledge. Our final example is a New Keynesian DSGE model with zero lower bound (Guerrieri and Iacoviello 2015). Solving New Keynesian DSGE models have been studied frequently in the literature, such as Woodford (2003), Del Negro et al. (2007), Smets and Wouters (2007), Gali (2008), Maliar and Maliar (2015), Fernández-Villaverde et al. (2015), and Guerrieri and Iacoviello (2015). Our results show that NLCEQ can easily solve competitive equilibrium problems with occasionally binding constraints. ${ }^{7}$

The paper is organized as follows. Section 2 introduces the NLCEQ method. Sections 3-6 apply NLCEQ to respectively solve multi-country RBC problems, a RBC model with a constraint on investment, a dynamic stochastic model of food and clean energy, and a New Keynesian DSGE model with zero lower bound. Section 7 concludes.

\section{NLCEQ Method}

An infinite horizon stochastic optimal decision-making problem can be expressed by the following general model:

$$
\begin{array}{r}
V\left(\mathbf{x}_{0}\right)=\max _{\mathbf{a}_{t} \in \mathcal{D}\left(\mathbf{x}_{t}\right)} \mathbb{E}\left\{\sum_{t=0}^{\infty} \beta^{t} u\left(\mathbf{x}_{t}, \mathbf{a}_{t}\right)\right\}, \\
\text { s.t. } \mathbf{x}_{t+1}=g\left(\mathbf{x}_{t}, \mathbf{a}_{t}, \varepsilon_{t}\right),
\end{array}
$$

where $\mathbf{x}_{t} \in \mathbb{R}^{d}$ is a state vector process with an initial state $x_{0}$ (each state variable could be either continuous or discrete), $\mathbf{a}_{t} \in \mathbb{R}^{n}$ is the vector of

\footnotetext{
${ }^{7}$ In this paper, we use GAMS (McCarl et al. 2011) code for all examples except for high-dimensional problems in Subsection 3.4. The NLCEQ method can also easily be implemented in other programming languages like MATLAB or Dynare (Adjemian et al. 2011). We use CONOPT (Drud 1996) as the optimization solver in our GAMS code, run them on one $3.5 \mathrm{GHz}$ Intel processor, and get the solution in seconds/minutes for each case. For high-dimensional problems in Subsection 3.4, we use Fortran code and SNOPT (Gill et al. 2005) as the optimization solver, implement parallelism on a supercomputer, and then get solutions in minutes.
} 
action variables at time $t, \varepsilon_{t}$ is a serially uncorrelated random vector process with identical and independent distributions across time (for simplicity, we assume that the mean or median of $\varepsilon_{t}$ is zero), $u(\mathbf{x}, \mathbf{a})$ is a utility function, $g(\mathbf{x}, \mathbf{a}, \varepsilon)$ is the stochastic law of motion for the state variable vector $\mathbf{x}, \beta$ is the discount factor $(0<\beta<1), \mathcal{D}\left(\mathbf{x}_{t}\right)$ is a feasible set of action $\mathbf{a}_{t}$, and $\mathbb{E}\{\cdot\}$ is the expectation operator. Here, $g$ is a general transition law of the vector of state variables, but some elements of the state variable vector $\mathbf{x}$ could be exogenous or have a deterministic transition law independent of $\varepsilon$.

To solve the problem (1), value function iteration is often used by solving the following Bellman equation backwards:

$$
\begin{gathered}
V_{t}\left(\mathbf{x}_{t}\right)=\max _{\mathbf{a}_{t} \in \mathcal{D}\left(\mathbf{x}_{t}\right)} u\left(\mathbf{x}_{t}, \mathbf{a}_{t}\right)+\beta \mathbb{E}\left\{V_{t+1}\left(\mathbf{x}_{t+1}\right)\right\}, \\
\text { s.t. } \mathbf{x}_{t+1}=g\left(\mathbf{x}_{t}, \mathbf{a}_{t}, \varepsilon_{t}\right) .
\end{gathered}
$$

Numerical implementation of value function iteration can be challenging for a number of reasons. It requires choosing an appropriate approximation domain for the state variables, which can be way wider than the one we are interested in, because of the stochasticity in the transition law of the states. In particular, when $\epsilon_{t}$ has an infinite support, this may lead to an infinite support for $\mathbf{x}_{t+1}$ so that we have to use some remedies like truncation methods. Their impact on the solution is, however, hard to be measured. Moreover, a wider domain requires a higher-degree approximation for the value functions, and then requires more time for an optimization solver to find the optimal solution of the Bellman equation. In addition, in the presence of multiple uncertainties, the integration part of the Bellman equation can be very time-consuming, and may even become infeasible, to get a good accuracy for high-dimensional integration. Finally, many problems have occasionally binding constraints which lead to kinks in value functions, a big challenge for multi-dimensional value function approximation. ${ }^{8}$

\footnotetext{
${ }^{8}$ Cai and Judd (2012) propose a rational spline interpolation method for value function approximation so that the value function iteration is stable and accurate for the problems
} 
Algorithm 1 Nonlinear Certainty Equivalent Approximation Method for Infinite-horizon Stochastic Dynamic Programming Problems

Step 1. Transformation step. Transform the infinite-horizon stochastic problem into a finite horizon deterministic optimal decision-making problem:

$$
\begin{gathered}
\widetilde{V}\left(\mathbf{x}_{0}\right)=\max _{a_{t} \in \mathcal{D}\left(\mathbf{x}_{t}\right)} \sum_{t=0}^{T-1} \beta^{t} u\left(\mathbf{x}_{t}, \mathbf{a}_{t}\right)+\beta^{T} \widetilde{V}_{T}\left(\mathbf{x}_{T}\right), \\
\text { s.t. } \mathbf{x}_{t+1}=g\left(\mathbf{x}_{t}, \mathbf{a}_{t}, 0\right)
\end{gathered}
$$

where $\widetilde{V}_{T}$ is a terminal value function given by an initial guess of the value function $V$.

Step 2. Optimization step. Choose a set of approximation nodes, $\mathbb{X}=\left\{\mathbf{x}_{0}^{j}\right.$ : $1 \leq j \leq m\} \subset \mathbb{R}^{d}$, and compute $v^{j}=\widetilde{V}\left(\mathbf{x}_{0}^{j}\right)$ and its corresponding optimal initial action $\mathbf{a}_{0}^{j} \in \mathbb{R}^{n}$ using an optimization solver to solve (3), for each $\mathbf{x}_{0}^{j} \in \mathbb{X}, 1 \leq j \leq m$.

Step 3. Approximation step. Using an appropriate approximation method, such that $\hat{V}\left(\mathbf{x}_{0} ; \mathbf{b}_{v}\right)$ approximates $\left\{\left(\mathbf{x}_{0}^{j}, v^{j}\right): 1 \leq j \leq m\right\}$ data and a vector of functions $\hat{\mathbf{P}}\left(\mathbf{x}_{0} ; \mathbf{b}_{a}\right)$ approximates $\left\{\left(\mathbf{x}_{0}^{j}, \mathbf{a}_{0}^{j}\right): 1 \leq j \leq m\right\}$, i.e., $v^{j} \approx \hat{V}\left(\mathbf{x}_{0}^{j} ; \mathbf{b}_{v}\right)$ and $\mathbf{a}_{0}^{j} \approx \hat{\mathbf{P}}\left(\mathbf{x}_{0}^{j} ; \mathbf{b}_{a}\right)$ for all $\mathbf{x}_{0}^{j} \in \mathbb{X}$, where $\mathbf{b}_{v}$ and $\mathbf{b}_{a}$ are vectors of parameters.

However, in many cases it is acceptable to obtain a solution to the problem (1) with less demanding accuracy. For these cases, below we propose a simple and fast nonlinear certainty equivalent (NLCEQ) approximation method (Algorithm 1) to obtain the value function $V$ and corresponding optimal decision rules.

NLCEQ is a natural extension of the certainty equivalent approximation idea that the locally accurate linearization (log-linearization) method implements, but it solves the deterministic optimization problems to find approximate values of value/policy functions at pre-specified state nodes and with kinks, but it applies to problems with only one continuous state variable. 
then uses global nonlinear approximation methods to get the approximate value/policy functions, so NLCEQ is a globally accurate method. Because (3) is a convex optimization problem for most of dynamic programming problems in economics, NLCEQ is stable and can work well for problems with occasionally binding constraints. Stability of the NLCEQ algorithm ensures that solution accuracy is little changed by variations in model parameter values (we illustrated this in examples of Subsection 3.2). ${ }^{9}$

If there is no uncertainty in the underlying problem, the NLCEQ method gives us a very accurate value and policy function for large enough $T$. For the stochastic problems, NLCEQ can give an estimate of the value/policy functions, which can be subsequently employed in the economic analysis, such as impulse function analysis and sensitivity analysis. To obtain more accurate approximation, if necessary, we can use the solutions of NLCEQ, $\hat{V}\left(\mathbf{x}_{0} ; \mathbf{b}_{v}\right)$ and $\hat{\mathbf{P}}\left(\mathbf{x}_{0} ; \mathbf{b}_{a}\right)$, as the initial guess for the value/policy functions, and then apply other more accurate methods like numerical value function iteration (Cai and Judd 2014). When there is some freedom in choosing $T$, $\widetilde{V}_{T}$, approximation nodes, and approximation methods, a criterion to choose them is that global errors defined in a way like in equation (19) are small (note that global errors are always no less than Euler errors). We will discuss the steps in more details below. ${ }^{10}$

After we get the optimal policy functions $\hat{\mathbf{P}}\left(\mathbf{x}_{0} ; \mathbf{b}_{a}\right)$, it is easy to do a forward simulation: with a given initial state $\mathbf{x}_{0}$ and one simulation path $\varepsilon_{t}$, we use $\mathbf{a}_{t}=\hat{\mathbf{P}}\left(\mathbf{x}_{t} ; \mathbf{b}_{a}\right)$ to get $\mathbf{x}_{t+1}=g\left(\mathbf{x}_{t}, \mathbf{a}_{t}, \varepsilon_{t}\right)$ for any time $t=0,1,2, \ldots$. That is, in the simulation process, we do not need to repeatedly apply NLCEQ or solve its optimization problem (3); instead we only need to use the solved policy functions $\hat{\mathbf{P}}\left(\mathbf{x} ; \mathbf{b}_{a}\right)$ while making sure that $\mathbf{x}_{t}$ is located inside

\footnotetext{
${ }^{9} \mathrm{~A}$ standard way to avoid any problems with model calibration and sensitivity analysis is to choose a wide approximation domain, so that changing calibrated parameter values does not push state variables outside the approximation domain, and choose large enough $T$ so that the terminal value functions do not have a significant effect on the solution.

${ }^{10}$ For a more complete and general discussion on approximation and optimization in solving dynamic stochastic problems, see Judd (1998) and Miranda and Fackler (2002).
} 
the approximation domain. ${ }^{11}$ We can do an impulse response analysis in a similar way.

\subsection{Transformation}

In the transformation step of the NLCEQ method, it is usually straightforward to obtain deterministic transition laws for continuous state variables. For example, if an exogenous state $\theta_{t}$ has a transition law: $\ln \left(\theta_{t+1}\right)=$ $\rho \ln \left(\theta_{t}\right)+\sigma \epsilon_{t+1}$, where $\epsilon_{t+1} \sim \mathcal{N}(0,1)$ enters linearly into the law of motion of the exogenous state, then a simple transformation is to set $\ln \left(\theta_{t+1}\right)=\rho \ln \left(\theta_{t}\right)$. A more general choice of the transformation is $\ln \theta_{t+1}=\rho \ln \theta_{t}-f(\sigma)$ with function $f$ chosen in such way that the deterministic $u\left(\mathbf{x}_{t}, \mathbf{a}_{t}\right)$ is close to the expectation of stochastic utility, i.e, the deterministic $u\left(\mathbf{x}_{t}, \mathbf{a}_{t}\right)$ is nearly a certainty equivalent of its stochastic version. One example of such transformation is shown in Subsection 4.3, where we obtain more accurate solution by choosing $\ln \left(\theta_{t+1}\right)=\rho \ln \left(\theta_{t}\right)-0.5 \sigma^{2}$ with $\sigma=0.05$. Using this general transformation, we can deal with problems such as stochastic volatility (see e.g., Caldara et al. 2012).

To choose the terminal value function, a typical way is to assume that it reaches an equilibrium at $T$, that is, the vector of control variables is chosen to be $\mathbf{a}_{T}^{*}$ so that next-period state is equal to current state $\mathbf{x}_{T}$, and then we let $\widetilde{V}_{T}\left(\mathbf{x}_{T}\right)=u\left(\mathbf{x}_{T}, \mathbf{a}_{T}^{*}\right) /(1-\beta)$. We use this in our multi-country RBC examples. Another potential way is to use a second-order perturbation as the terminal value function, but we do not apply it in this paper because we want to focus on NLCEQ only, without hybrid algorithms. The truncation time, $T$, depends on the terminal value function and the discount factor. If the terminal value function is close to the true value function, then $T$ could

\footnotetext{
${ }^{11}$ That is, we have to choose an appropriate approximation domain in the optimization step, so that it is wide enough to contain simulated future states. This can be done in an iterative way: first guess a wider approximation domain, and then use the NLCEQ solution over it to do simulation: if the simulated states locate in a much more narrow domain, choose the narrower domain to re-run NLCEQ algorithm for a more accurate solution.
} 
be small, for example, we choose $T=20$ for some large-dimensional multicountry RBC examples in Section 3; otherwise, $T$ could be chosen such that $\beta^{T}<10^{-4}$ if it is hard to find a terminal value function close to the true value function. For example, we choose $T=200$ in the example with $\beta=0.95$ in Section 5 such that $\beta^{T}=3.5 \times 10^{-5}$.

We can also apply NLCEQ to problems with a discrete stochastic state $\theta_{t}$ by replacing it by its expected value conditional on its initial value, i.e., $\mathbb{E}\left\{\theta_{t} \mid \theta_{0}\right\}$. For example, let $\theta_{t}$ be an exogenous Markov chain with $k$ possible values, $\left\{\vartheta_{1}, \ldots, \vartheta_{k}\right\}$, and let $P$ be its $k \times k$ transition matrix, where its $(i, j)$ element represents the probability of $\theta_{t+1}=\vartheta_{i}$ conditional on $\theta_{t}=\vartheta_{j}$. If the initial-time value of $\theta_{t}$ is $\vartheta_{i}$ (i.e., $\theta_{0}=\vartheta_{i}$ ), then we know that its unconditional probability vector at time $t$ is $p_{t, i}=P^{t} \mathbf{e}_{i}$, where $\mathbf{e}_{i}$ is the column vector with 1 at the $i$-th element and 0 everywhere else. Thus, in the transformation step, we set the transformed deterministic value for $\theta_{t}$ as its expected value, $\sum_{j=1}^{k} p_{t, i, j} \vartheta_{j}$, conditional on its initial value $\theta_{0}=\vartheta_{i}$, where $p_{t, i, j}$ is the $j$-th element of the vector $p_{t, i}$. Our example in Section 5 has a discrete stochastic state and applies this method.

\subsection{Optimization}

In the NLCEQ method, the optimization step will be time-consuming for high-dimensional problems, but they can be naturally parallelized across the approximation nodes, as every node corresponds to one optimization problem, which is independent of the others. Moreover, each optimization problem has a sparsity structure: the action variables and state variables at time $t$ are only connected with the state variables at $t-1$ and $t+1$, that is, it has the block-wise tridiagonal pattern in the constraints. We employ this sparsity in optimization solvers like the one we used in our high-dimensional multicountry RBC examples, SNOPT (Gill et al. 2005), so that each optimization 
problem can be solved more efficiently. ${ }^{12}$

\subsection{Approximation}

For low-dimensional problems, we can use a variety of approximation methods like, for example, multi-dimensional Chebyshev polynomial approximation (see Appendix A). However, one advantage of NLCEQ is that it can be applied to large-dimensional problems. For large-dimensional problems, we will use sparse grid approximation methods. For example, in our largedimensional examples, we employ Smolyak grid points as the approximation nodes and Chebyshev-Smolyak polynomials as the approximation method (Smolyak 1963 and Malin et al. 2011). ${ }^{13}$ Moreover, we can also implement adaptive sparse grid methods (Brumm and Scheidegger 2014) in NLCEQ.

After we get the approximated value/policy functions, $\widehat{V}\left(\mathbf{x}_{0} ; \mathbf{b}_{v}\right)$ and $\widehat{\mathbf{P}}\left(\mathbf{x}_{0} ; \mathbf{b}_{a}\right)$, it is essential to estimate their errors to the "true" solution so we know whether NLCEQ gives an acceptable solution. In our examples below we implement the unit-free Euler error measure. We also compute approximation errors for the approximation functions. That is, we choose a set of out-of-sample points, $\widehat{\mathbb{X}}=\left\{\widehat{\mathbf{x}}_{0}^{j}: 1 \leq j \leq \widehat{m}\right\} \subset \mathbb{R}^{d}$, and compute $\widehat{v}^{j}=\widetilde{V}\left(\widehat{\mathbf{x}}_{0}^{j}\right)$ and its corresponding optimal initial action $\widehat{\mathbf{a}}_{0}^{j} \in \mathbb{R}^{n}$ using op-

\footnotetext{
${ }^{12}$ Optimization step of NLCEQ algorithm can be employed with a variety of modern nonlinear optimization solvers, such as e.g., SNOPT (Gill et al. 2005), CONOPT (Drud 1996), and KNITRO (Byrd et al. 2006). These solvers are also freely available at the NEOS server (Czyzyk et al. 1998; Gropp and Moré 1997) with two popular high-level modeling languages: GAMS (McCarl et al. 2011) and AMPL (Fourer et al. 2003). If the code is written in MATLAB, it can call its internal optimization routine, fmincon, or an external solver such as KNITRO.

${ }^{13}$ Smolyak polynomials do not preserve shape of value functions, so using them in standard value function iteration can easily make it fail because the optimization problem in the Bellman equation becomes a non-concave/non-convex problem, thus it is very challenging to find the global maximizer by a standard optimization solver. See Cai and Judd $(2013,2015)$ for discussion about the importance of shape preservation in numerical DP. However, with NLCEQ algorithm we do not need to use the approximate value functions in the objective of an optimization problem, so it does not face the shape-preservation challenge while the value function iteration does.
} 
timization solver to solve (3), for each $\widehat{\mathbf{x}}_{0}^{j} \in \widehat{\mathbb{X}}, 1 \leq j \leq \widehat{m}$. Using these $\widehat{v}^{j}$, we compute the approximation errors in the $\mathcal{L}^{\infty}$ or $\mathcal{L}^{1}$ norm for the value function with the following formulas:

$$
\begin{gathered}
\widehat{E}_{\mathcal{L}^{\infty}}=\max _{1 \leq j \leq \widehat{m}} \frac{\left|\widehat{v}^{j}-\widehat{V}\left(\widehat{\mathbf{x}}_{0}^{j} ; \mathbf{b}_{v}\right)\right|}{1+\left|\widehat{v}^{j}\right|} \\
\widehat{E}_{\mathcal{L}^{1}}=\frac{1}{\widehat{m}} \sum_{1 \leq j \leq \widehat{m}} \frac{\left|\widehat{v}^{j}-\widehat{V}\left(\widehat{\mathbf{x}}_{0}^{j} ; \mathbf{b}_{v}\right)\right|}{1+\left|\widehat{v}^{j}\right|}
\end{gathered}
$$

Similarly, we can compute approximation errors for the policy functions. In our examples, we let $\widehat{\mathbb{X}}$ be a set of 1000 points uniformly and randomly drawn in the approximation domain. Note that the computation of $\widehat{v}^{j}=$ $\widetilde{V}\left(\widehat{\mathbf{x}}_{0}^{j}\right)$ and its corresponding $\widehat{\mathbf{a}}_{0}^{j}$ can be parallelized naturally together with the optimization step of NLCEQ.

\subsection{NLCEQ Method for Competitive Equilibrium}

Algorithm 1 describes the NLCEQ method for social planner's decisionmaking problems, but it can also be modified for solving competitive equilibrium. Similar to the transformation step of Algorithm 1, we first remove the stochasticity of models by replacing those shocks by their mean or median. For the transformed deterministic problem, its equilibrium solution should satisfy a set of equations (including the deterministic version of Euler equations, deterministic transition laws of states, market clearing conditions, and other first-order conditions):

$$
\mathbf{F}\left(\mathbf{x}_{t}, \mathbf{a}_{t}, \mathbf{x}_{t+1}, \mathbf{a}_{t+1}\right)=0,, \quad t=0,1,2, \ldots
$$

where $\mathbf{x}_{t}$ is the state vector, and $\mathbf{a}_{t}$ is the action vector that should satisfy constraints $\mathbf{a}_{t} \in \mathcal{D}\left(\mathbf{x}_{t}\right)$. If there are occasionally binding constraints, then 
Algorithm 2 Nonlinear Certainty Equivalent Approximation Method for Competitive Equilibrium

Step 1. Transformation step. Transform the stochastic problem into a finitehorizon deterministic system (5).

Step 2. Optimization step. Choose a set of approximation nodes, $\mathbb{X}=\left\{\mathbf{x}_{0}^{j}\right.$ : $1 \leq j \leq m\} \subset \mathbb{R}^{d}$. For each $\mathbf{x}_{0}^{j} \in \mathbb{X}$, solve (5) and get its corresponding optimal initial action with $\mathbf{a}_{0}^{j}$.

Step 3. Approximation step. Use an appropriate approximation method, such that $\hat{\mathbf{P}}\left(\mathbf{x}_{0} ; \mathbf{b}_{a}\right)$ approximates $\left\{\left(\mathbf{x}_{0}^{j}, \mathbf{a}_{0}^{j}\right): 1 \leq j \leq m\right\}$, where $\mathbf{b}_{a}$ is a vector of parameters.

the arguments of $\mathbf{F}$ should also contain corresponding Lagrange multipliers that we omit below without loss of generality. Moreover, we know that its state and control variables will converge to its steady values $\left(\mathbf{x}_{s s}, \mathbf{a}_{s s}\right)$ as time goes to infinity, i.e.,

$$
\mathbf{x}_{\infty}=\mathbf{x}_{s s}, \mathbf{a}_{\infty}=\mathbf{a}_{s s} .
$$

To solve the above infinite-horizon system (4), we approximate it as the following minimization problem with a finite horizon and a given initial state $\mathbf{x}_{0}^{j}$ :

$$
\begin{aligned}
\min _{\mathbf{a}_{t} \in \mathcal{D}\left(\mathbf{x}_{t}\right)} & \left\|\mathbf{x}_{T}^{\text {Endo }}-\mathbf{x}_{s s}^{\text {Endo }}\right\|+\left\|\mathbf{a}_{T}-\mathbf{a}_{s s}\right\| \\
\text { s.t. } & \mathbf{F}\left(\mathbf{x}_{t}, \mathbf{a}_{t}, \mathbf{x}_{t+1}, \mathbf{a}_{t+1}\right)=0, \quad t=0,1, \ldots, T-1, \\
& \mathbf{x}_{0}=\mathbf{x}_{0}^{j},
\end{aligned}
$$

where $\|\cdot\|$ is a norm and $\mathbf{x}^{\text {Endo }}$ represents the endogenous state variables. By sweeping over the approximation nodes of $\mathbf{x}_{0}^{j}$, we can construct the approximation of policy functions over the state space. Algorithm 2 summarizes the NLCEQ method for solving competitive equilibrium.

Algorithm 2 yields very accurate solution of a deterministic competitive 
equilibrium problem for large enough T. Similar to Algorithm 1, Algorithm 2 is also stable and efficient, and can be naturally parallelized in its optimization step, so that it can solve large-dimensional problems using sparse grid approximation methods.

Algorithm 2 can also be applied to solve social planner's stochastic dynamic programming problems, but Algorithm 1 is easier to implement as it does not require formulating the first-order conditions. Therefore, in the examples of this paper, we will use Algorithm 1 for solving social planner's problems and Algorithm 2 for computing competitive equilibrium. .

\section{Application to multi-country real business cycle model}

We apply NLCEQ to solve a multi-country real business cycle (RBC) model introduced in Den Haan et al. (2011). We assume that there are $N$ countries with a capital stock state vector $K_{t}=\left(K_{t, 1}, \ldots, K_{t, N}\right)$ and a productivity state vector $\theta_{t}=\left(\theta_{t, 1}, \ldots, \theta_{t, N}\right)$ at the beginning of period $t$. For the $j$-th country, its production at time $t$ is given by $\theta_{t, j} f\left(K_{t, j}, \ell_{t, j}\right)$, where $\ell_{t, j}$ is labor supply and $f$ is the Cobb-Douglas production function

$$
f\left(K_{t, j}, \ell_{t, j}\right)=A\left(K_{t, j}\right)^{\alpha}\left(\ell_{t, j}\right)^{1-\alpha}
$$

where $\alpha$ is the expenditure share of capital in the production and $A$ is the productivity parameter. The law of motion of capital is:

$$
K_{t+1, j}=(1-\delta) K_{t, j}+I_{t, j}
$$

where $I_{t, j}$ is investment and $\delta$ is the depreciation rate of capital. The law of motion of productivity is exogenous:

$$
\ln \left(\theta_{t+1, j}\right)=\rho \ln \left(\theta_{t, j}\right)+\sigma\left(\epsilon_{t+1, j}+\varepsilon_{t+1}\right)
$$


where $\epsilon_{t, j}, \varepsilon_{t} \sim$ i.i.d. $\mathcal{N}(0,1)$ are a country specific shock and a worldwide shock, respectively.

The $j$-th country has an instantaneous utility

$$
u_{j}\left(c_{t, j}, \ell_{t, j}\right)=\frac{\left(c_{t, j}\right)^{1-\frac{1}{\gamma_{j}}}}{1-\frac{1}{\gamma_{j}}}-B_{j} \frac{\left(\ell_{t, j}\right)^{1+\frac{1}{\eta_{j}}}}{1+\frac{1}{\eta_{j}}}
$$

where $c_{t, j}$ is consumption, $\gamma_{j}$ is the inter-temporal elasticity of substitution, $\eta_{j}$ is the Frisch elasticity of labor supply, and $B_{j}=(1-\alpha) A^{\left(\gamma_{j}-1\right) / \gamma_{j}}$ is the relative weight of consumption and leisure in the welfare.

We want to solve a social planner's problem with an aggregate utility $U\left(c_{t}, \ell_{t}\right)$, which is the weighted sum of the instantaneous utilities of all countries, i.e.,

$$
U\left(c_{t}, \ell_{t}\right)=\sum_{j=1}^{N} \tau_{j} u_{j}\left(c_{t, j}, \ell_{t, j}\right)
$$

where $\tau_{j}=A^{1 / \gamma_{j}}$ are Negishi weights, $c_{t}=\left(c_{t, 1}, \ldots, c_{t, N}\right)$ and $\ell_{t}=\left(\ell_{t, 1}, \ldots, \ell_{t, N}\right)$. Let $\beta$ be the discount factor, and let $\Gamma_{t, j}$ be an adjustment cost:

$$
\Gamma_{t, j} \equiv \frac{\phi}{2} K_{t, j}\left(\frac{I_{t, j}}{K_{t, j}}-\delta\right)^{2}
$$

with $\phi$ as the intensity of the friction. The social planner problem then becomes

$$
\max _{c, \ell, I} \mathbb{E}\left(\sum_{t=0}^{\infty} \beta^{t} U\left(c_{t}, \ell_{t}\right)\right)
$$

subject to (7), and the following aggregate world resource constraint:

$$
\sum_{j=1}^{N}\left(c_{t, j}+I_{t, j}-\delta K_{t, j}\right)=\sum_{j=1}^{N}\left(\theta_{t, j} f\left(K_{t, j}, \ell_{t, j}\right)-\Gamma_{t, j}\right)
$$

Therefore, the problem has $2 N$ state variables, $K_{t}$ and $\theta_{t}$, and $3 N$ control variables, $c_{t}, \ell_{t}$, and $I_{t}$. 
In our example, we set $A=(1-\beta) /(\alpha \beta)$ and use the parameter values in Juillard and Villemot (2011) as the default. That is, we set $\beta=0.99$, $\alpha=0.36, \delta=0.025, \rho=0.95, \phi=0.5$, and $\sigma=0.01$, as the default values. Since the optimal solution depends only on the states and not the time $t$, we use $(K, \theta)$ to denote current states by omitting the subscript $t$, and use $\left(K^{+}, \theta^{+}\right)$to denote next-period states. We are interested in the policy solutions over the domain of the state variables $(K, \theta) \in[0.7,1.3]^{2 N}$.

\section{$3.1 \quad$ Error Measure}

For a given current state vector $(K, \theta)$, the first-order conditions of the $\mathrm{RBC}$ model (11) tell us that the optimal policy $(c, \ell, I)$ should satisfy

$$
\begin{gathered}
\frac{\partial u_{j}}{\partial c}\left(c_{j}, \ell_{j}\right) \tau_{j}=\frac{\partial u_{j^{\prime}}}{\partial c}\left(c_{j^{\prime}}, \ell_{j^{\prime}}\right) \tau_{j^{\prime}}, \quad j^{\prime} \neq j, \\
\frac{\partial u_{j}}{\partial \ell}\left(c_{j}, \ell_{j}\right)=-\frac{\partial u_{j}}{\partial c}\left(c_{j}, \ell_{j}\right) \theta_{j} \frac{\partial f}{\partial \ell}\left(K_{j}, \ell_{j}\right),
\end{gathered}
$$

and the following $N$ Euler equations:

$$
\frac{\partial u_{j}}{\partial c}\left(c_{j}, \ell_{j}\right) \omega_{j}=\mathbb{E}\left\{\beta \frac{\partial u_{j}}{\partial c}\left(c_{j}^{+}, \ell_{j}^{+}\right)\left[\pi_{j}^{+}+\theta_{j}^{+} \frac{\partial f}{\partial K}\left(K_{j}^{+}, \ell_{j}^{+}\right)\right]\right\}
$$

for $j=1, \ldots, N$. Here,

$$
\begin{aligned}
\omega_{j} & \equiv 1+\phi\left(\frac{I_{j}}{K_{j}}-\delta\right), \\
\pi_{j}^{+} & \equiv 1+\frac{\phi}{2}\left(\frac{I_{j}^{+}}{K_{j}^{+}}-\delta\right)\left(2-\delta+\frac{I_{j}^{+}}{K_{j}^{+}}\right),
\end{aligned}
$$

and $\left(c^{+}, \ell^{+}, I^{+}\right)$is the optimal policy in the next period.

We use NLCEQ to get the estimate of the optimal policy functions of the problem (11): $C_{j}(K, \theta)$ for consumption, $L_{j}(K, \theta)$ for labor supply, $\mathcal{I}_{j}(k, \theta)$ 
for investment, and $\mathcal{K}_{j}^{+}(K, \theta)=(1-\delta) K+\mathcal{I}_{j}(K, \theta)$ for the next-period capital. Thus, for any $(K, \theta)$, we can compute $c_{j}=C_{j}(K, \theta), \ell_{j}=L_{j}(K, \theta)$, $I_{j}=\mathcal{I}_{j}(K, \theta), K_{j}^{+}=\mathcal{K}_{j}^{+}(K, \theta), c_{j}^{+}=C_{j}\left(K^{+}, \theta^{+}\right), \ell_{j}^{+}=L_{j}\left(K^{+}, \theta^{+}\right), I_{j}^{+}=$ $\mathcal{I}_{j}\left(K^{+}, \theta^{+}\right)$, and then $\omega_{j}$ and $\pi_{j}^{+}$from the equations (16) and (17).

Therefore, for a given $(K, \theta)$, we can compute the following unit-free Euler error:

$$
E_{1}(K, \theta)=\max _{1 \leq j \leq N}\left|\mathbb{E}\left\{F_{j}\left(K, \theta, \theta^{+}\right)\right\}-1\right|,
$$

with

$$
F_{j}\left(K, \theta, \theta^{+}\right) \equiv \frac{\beta \frac{\partial u_{j}}{\partial c}\left(c_{j}^{+}, \ell_{j}^{+}\right)}{\frac{\partial u_{j}}{\partial c}\left(c_{j}, \ell_{j}\right) \omega_{j}}\left[\pi_{j}^{+}+\theta_{j}^{+} \frac{\partial f}{\partial K}\left(K_{j}^{+}, \ell_{j}^{+}\right)\right] .
$$

Moreover, the unit-free errors for the intratemporal-choice conditions (13) and (14) are also available:

$$
\begin{gathered}
E_{2}(K, \theta)=\max _{2 \leq j \leq N}\left|\frac{\frac{\partial u_{j}}{\partial c}\left(c_{j}, \ell_{j}\right) \tau_{j}}{\frac{\partial u_{1}}{\partial c}\left(c_{1}, \ell_{1}\right) \tau_{1}}-1\right|, \\
E_{3}(K, \theta)=\max _{1 \leq j \leq N}\left|\frac{\frac{\partial u_{j}}{\partial c}\left(c_{j}, \ell_{j}\right) \theta_{j} \frac{\partial f}{\partial \ell}\left(K_{j}, \ell_{j}\right)}{\frac{\partial u_{j}}{\partial \ell}\left(c_{j}, \ell_{j}\right)}+1\right| .
\end{gathered}
$$

The unit-free error for the resource constraint is given by

$$
E_{4}(K, \theta)=\left|\frac{\sum_{j=1}^{N}\left(c_{j}+I_{j}-\delta K_{j}+\Gamma_{j}\right)}{\sum_{j=1}^{N}\left(\theta_{j} f\left(K_{j}, \ell_{j}\right)\right)}-1\right| .
$$

Using the above errors for the first-order conditions and the resource constraint, we compute the following global $\mathcal{L}^{\infty}$ error on a domain of $(K, \theta)$, denoted $\mathscr{D}$, to measure the accuracy of our solution:

$$
\mathcal{E}=\max _{(K, \theta) \in \mathscr{D}}\left\{\max _{1 \leq i \leq 4} E_{i}(K, \theta)\right\} .
$$

Note that the estimated policy functions $C_{j}, L_{j}$ and $\mathcal{I}_{j}$ should be defined not only in the domain of $(K, \theta) \in \mathscr{D}$ (in our examples, we let $\mathscr{D}=$ 
$\left.[0.7,1.3]^{2 N}\right)$, but also in a wider domain for $\left(K^{+}, \theta^{+}\right)$. Therefore, in order to get the Euler errors $E_{1}$, we should apply NLCEQ in a wider domain than what we are interested. In our examples, we choose a domain $[0.5,1.5]^{2 N}$ for approximating NLCEQ policy functions, and then estimate the global error in $[0.7,1.3]^{2 N}$. In addition, we could have $E_{2}$ and $E_{3}$ (and even $E_{4}$ ) to be zero: for any $(K, \theta)$, we let $c_{1}=C_{1}(K, \theta)$ and compute other $c_{j}$ from the equations (13) instead of letting $c_{j}=C_{j}(K, \theta)$, and then compute $\ell_{j}$ from (14) instead of letting $\ell_{j}=L_{j}(K, \theta)$. This method may obtain a smaller global error as it has smaller approximation errors from $C_{j}$ and $L_{j}$. But this method may require solving a complicated system of nonlinear equations, so we do not apply it in this paper for more generality.

To compute the Euler error $E_{1}(K, \theta)$ for a given $(K, \theta)$, we estimate the integration in (18) using Monte Carlo simulation method with 10,000 points randomly drawn from the distribution of $\theta^{+}$(when $N \leq 4$, we can use the Gauss-Hermite quadrature rule with 7 quadrature nodes in each dimension for a faster run). Since the standard deviation of $F_{j}\left(k, \theta, \theta^{+}\right)$is around the size of $\sigma$ in all of our cases, the accuracy of the numerical integration is about $10^{-4}$, acceptable for measuring the errors of NLCEQ. In our results, the global error $\mathcal{E}$ is estimated by the maximal value of $\max _{1 \leq i \leq 4} E_{i}(K, \theta)$ among 10,000 randomly and uniformly drawn points $(K, \theta)$ in the domain $[0.7,1.3]^{2 N}$. This is time-consuming for high-dimensional problems, but it can also be parallelized naturally. For all of our examples, we computed the standard error of the estimated expectation, and found that the standard error is one or two orders of magnitude smaller than the Euler error, so the numerical integration error is negligible in our computation of the Euler error.

\subsection{Examples for Accuracy Test}

We first test NLCEQ for its accuracy for the two-country real business cycle problem (i.e., $N=2$ ), which has four continuous state variables: two capital 
stocks and two productivity levels. In the transformation step of NLCEQ (Algorithm 1), we choose $T=50$ and the problem becomes

$$
\widetilde{V}\left(K_{0}, \theta_{0}\right)=\max _{c, \ell, I} \sum_{t=0}^{T-1} \beta^{t} U\left(c_{t}, \ell_{t}\right)+\beta^{T} \widetilde{V}_{T}\left(K_{T}, \theta_{T}\right)
$$

subject to (7) and (12) with a deterministic process of $\theta_{t}: \ln \left(\theta_{t+1, j}\right)=$ $\rho \ln \left(\theta_{t, j}\right)$. The terminal value function $\widetilde{V}_{T}(K, \theta)$ is given as $U\left(f\left(K, \ell^{*}\right), \ell^{*}\right) /(1-$ $\beta)$ with $\ell^{*}=(1, \ldots, 1)$.

In the NLCEQ method, we first use the tensor grid of Chebyshev nodes $(D+1$ nodes in each dimension) over the domain of the state variables, $[0.5,1.5]^{2 N}$, and then apply degree- $D$ complete Chebyshev polynomials in the approximation step. Since we will apply NLCEQ to high-dimensional problems using the level-l Smolyak points and Chebyshev-Smolyak polynomials (a subset of degree- $2^{l}$ complete Chebyshev polynomials) for approximation, we also try them in the low-dimensional problems to check their accuracy.

Our starting examples have symmetric model specification, that is, we let $\gamma_{j}$ be a constant $\gamma$, and let $\eta_{j}$ be a constant $\eta$. Table 1 lists the global errors in $\mathcal{L}^{\infty}$ norm over $[0.7,1.3]^{2 N}$ for the symmetric cases with $\beta \in\{0.99,0.95\}$, $\gamma \in\{0.25,0.5\}, \eta \in\{0.1,0.5\}$, and $\sigma=0.01 .^{14}$ From Table 1 , we see that degree-4 complete Chebyshev polynomials have the smallest global errors at $O\left(10^{-3}\right) .{ }^{15}$

${ }^{14}$ The range for $\theta,[0.7,1.3]^{N}$, is narrow: from $(8)$, if $\theta_{t, j}$ is inside the following range

$$
\left[\exp \left(\frac{-\sqrt{2} \sigma}{1-\rho}\right), \exp \left(\frac{\sqrt{2} \sigma}{1-\rho}\right)\right],
$$

which is close to $[0.7,1.3]$, then only when $\epsilon_{t+1, j}$ and $\varepsilon_{t+1}$ are simulated to let $\epsilon_{t+1, j}+\varepsilon_{t+1}$ be bounded in $[-\sqrt{2}, \sqrt{2}]$, we can make sure that $\theta_{t+1, j}$ is inside the same range. That is, if $\theta_{t, j}$ is at one end of the range, then it has about $16 \%$ probability that $\theta_{t+1, j}$ is outside of the range. Kollmann et al. (2011) also checked the errors of solutions of perturbation methods on the sphere in the state space centered at the steady state with a radius 0.3 .

${ }^{15}$ Since our solutions are independent of the value of $\sigma$ but the Euler errors $E_{1}$ depend on $\sigma$, we also checked the errors with $\sigma=0.02$. We found that the global errors of $\sigma=0.02$ are the same with the ones of $\sigma=0.01$ although the $\sigma$-related maximal Euler errors with 
Table 1: Global errors in $\mathcal{L}^{\infty}$ norm for two-country problems

\begin{tabular}{c|c|c|cc|cc}
\hline \multirow{3}{*}{$\beta$} & \multirow{3}{*}{$\gamma$} & & \multicolumn{4}{|c}{ Global Error $\mathcal{E}$} \\
\cline { 4 - 7 } & & & \multicolumn{2}{|c|}{ degree- $D$} & Chebyshev & \multicolumn{2}{l}{ level-l Smolyak } \\
\cline { 3 - 7 } & & $D=2$ & $D=4$ & $l=1$ & $l=2$ \\
\hline 0.99 & 0.25 & 0.1 & $2.4(-2)$ & $1.7(-3)$ & $5.3(-2)$ & $6.7(-3)$ \\
& & 0.5 & $2.1(-2)$ & $2.0(-3)$ & $6.5(-2)$ & $1.0(-2)$ \\
\cline { 3 - 7 } & 0.5 & 0.1 & $2.0(-2)$ & $1.3(-3)$ & $6.1(-2)$ & $5.3(-3)$ \\
& & 0.5 & $2.1(-2)$ & $1.1(-3)$ & $6.5(-2)$ & $6.1(-3)$ \\
\hline 0.95 & 0.25 & 0.1 & $2.8(-2)$ & $2.6(-3)$ & $5.1(-2)$ & $9.3(-3)$ \\
& & 0.5 & $1.8(-2)$ & $3.7(-3)$ & $7.0(-2)$ & $1.3(-2)$ \\
\cline { 3 - 7 } & 0.5 & 0.1 & $2.0(-2)$ & $1.5(-3)$ & $5.7(-2)$ & $5.6(-3)$ \\
& & 0.5 & $1.5(-2)$ & $1.7(-3)$ & $6.2(-2)$ & $8.7(-3)$ \\
\hline
\end{tabular}

Note: $\zeta(-j)$ represents $\zeta \times 10^{-j}$.

Table 2: Errors in $\mathcal{L}^{\infty}$ norm for two-country problems with $\sigma=0$

\begin{tabular}{c|ccc|ccc}
\hline & \multicolumn{3}{|c|}{ degree- $D$ Chebyshev } & \multicolumn{3}{c}{ level- $l$ Smolyak } \\
& $D=4$ & $D=6$ & $D=8$ & $l=2$ & $l=3$ & $l=4$ \\
\hline Euler Error & $4.2(-5)$ & $2.1(-5)$ & $2.1(-5)$ & $2.6(-4)$ & $2.7(-5)$ & $2.1(-5)$ \\
Global Error & $1.1(-3)$ & $5.6(-5)$ & $3.8(-6)$ & $5.9(-3)$ & $6.1(-4)$ & $7.3(-5)$ \\
\hline
\end{tabular}

Note: $\zeta(-j)$ represents $\zeta \times 10^{-j}$.

We also show that NLCEQ (Algorithm 1) can very accurately solve deterministic dynamic problems. Table 2 lists the maximal Euler errors and global errors in $\mathcal{L}^{\infty}$ norm over $[0.7,1.3]^{2 N}$ for the two-country problem with $\sigma=0$ and $\beta=0.99, \gamma=0.5$, and $\eta=0.5$ (the results are similar for other values of $(\beta, \gamma, \eta))$. As we seek higher accuracy and the only source of errors for deterministic problems comes from the truncation of infinite horizon and the value/policy function approximation, we use a large $T=200$ and high degree approximation. From Table 2, we see that the global errors are always larger than Euler errors so it is not good enough to use Euler errors as a criterion. Moreover, we see that our solution can reach five-digit accuracy in $\mathcal{L}^{\infty}$ for the deterministic problem.

$\sigma=0.02$ are a bit higher. 


\subsection{Comparison with log-linearization}

Log-linearization is the most popular method for solving dynamic stochastic models. It is also a certainty equivalent approximation method: it computes a log-linear approximation for the policy function of a deterministic problem and uses it in simulations of the stochastic model. While this may be acceptable for states close to the deterministic steady state, it is not likely to be a good approximation beyond a small neighborhood around the steady state. This is particularly relevant if the mean of the stochastic problem is not the deterministic steady state.

We next present one two-country example where we compare NLCEQ with log-linearization. We use an example with asymmetric model specification, by assuming $\gamma_{1}=0.25$ and $\eta_{1}=0.1$ for the first country, and $\gamma_{2}=1$ and $\eta_{2}=1$ for the second country, in their utility functions. The other parameters are set as their default values. We use the degree- 4 complete Chebyshev polynomials for approximation in NLCEQ.

For NLCEQ, the global $\mathcal{L}^{\infty}$ error, $\mathcal{E}$, is 0.0014 , which is similar to the examples in Table 1. Kollmann et al. (2011) report the errors of solutions from other methods for this asymmetric case (Model II with $N=2$ in their Table 4). The perturbation methods (order-1 or order-2) have large errors on the sphere in the state space centered at the steady state with a radius 0.3 (this sphere is inside our domain $[0.7,1.3]^{2 N}$ ). The $\mathcal{L}^{\infty}$ error of the loglinear approximation (i.e., the order-1 perturbation which is linear in $\log (K)$ and $\log (\theta)$ ) is 0.51 . Even its extended order-2 perturbation method (with quadratic polynomials in $\log (K)$ and $\log (\theta))$ has an $\mathcal{L}^{\infty}$ error equal to 0.21 . Thus, NLCEQ is far more accurate, up to two orders of magnitude higher, than log-linearization over the wider and more relevant domain. 
Table 3: Errors and running times for high-dimensional RBC problems

\begin{tabular}{|c|c|c|c|c|c|c|c|}
\hline$N$ & Level $l$ & $\begin{array}{l}\text { Num of } \\
\text { Points }\end{array}$ & $\begin{array}{l}\text { Num of } \\
\text { Cores }\end{array}$ & $T$ & $\begin{array}{l}\text { Max Euler } \\
\text { Error }\end{array}$ & $\begin{array}{l}\text { Global } \\
\text { Error }\end{array}$ & $\begin{array}{c}\text { Time } \\
\text { (minutes) }\end{array}$ \\
\hline \multirow[t]{4}{*}{10} & \multirow[t]{2}{*}{1} & \multirow[t]{2}{*}{41} & \multirow[t]{2}{*}{41} & 20 & $3.5(-3)$ & $2.4(-2)$ & 0.5 \\
\hline & & & & 50 & $3.6(-3)$ & $2.6(-2)$ & 0.4 \\
\hline & \multirow[t]{2}{*}{2} & \multirow[t]{2}{*}{841} & \multirow[t]{2}{*}{288} & 20 & $3.2(-3)$ & $4.8(-3)$ & 1.5 \\
\hline & & & & 50 & $7.1(-4)$ & $4.3(-3)$ & 1.4 \\
\hline \multirow[t]{5}{*}{20} & \multirow[t]{2}{*}{1} & \multirow[t]{2}{*}{81} & \multirow[t]{2}{*}{81} & 20 & $2.6(-3)$ & $1.9(-2)$ & 0.2 \\
\hline & & & & 50 & $2.0(-3)$ & $1.9(-2)$ & 1.3 \\
\hline & \multirow[t]{3}{*}{2} & \multirow[t]{3}{*}{3,281} & 352 & 20 & $2.1(-3)$ & $3.3(-3)$ & 1.7 \\
\hline & & & 352 & 50 & $5.8(-4)$ & $3.1(-3)$ & 13.5 \\
\hline & & & 3,281 & 50 & $5.8(-4)$ & $3.1(-3)$ & 1.6 \\
\hline \multirow[t]{5}{*}{50} & \multirow[t]{2}{*}{1} & \multirow[t]{2}{*}{201} & \multirow[t]{2}{*}{201} & 20 & $2.3(-3)$ & $1.8(-2)$ & 0.8 \\
\hline & & & & 50 & $1.9(-3)$ & $1.8(-2)$ & 5.7 \\
\hline & \multirow[t]{3}{*}{2} & \multirow[t]{3}{*}{20,201} & 2,048 & 20 & $1.5(-3)$ & $2.7(-3)$ & 8.3 \\
\hline & & & 2,048 & 50 & $3.5(-4)$ & $2.6(-3)$ & 58.1 \\
\hline & & & 20,201 & 50 & $3.5(-4)$ & $2.6(-3)$ & 8.6 \\
\hline 100 & 1 & 401 & 401 & 20 & $1.9(-3)$ & $1.8(-2)$ & 2.2 \\
\hline 200 & 1 & 801 & 801 & 20 & $1.6(-3)$ & $1.8(-2)$ & 8.0 \\
\hline
\end{tabular}

\subsection{Application to High-dimensional Problems}

In this subsection we use NLCEQ to solve the high-dimensional RBC problems with the default parameter values and $\gamma_{j} \equiv 0.25$ and $\eta_{j} \equiv 0.1$. We use the same transformed deterministic model (20) with $T=20$ or 50 for more countries, and use the level- $l$ Smolyak grid and Chebyshev-Smolyak polynomials for approximation.

Table 3 lists the Euler errors and global errors in $\mathcal{L}^{\infty}$ norm over $[0.7,1.3]^{2 N}$ and running times (in wall clock time) in minutes, for cases with the number of countries $N=10,20,50,100,200$ (the dimension of continuous state variables is $2 N)$. For example, for the case with $N=200$ countries and $T=20$, its maximal Euler error is 0.0016 and its global error is 0.018, and it is solved in only 8 minutes. 
We employ parallelism in a supercomputer. Table 3 lists the numbers of approximation points (level-l Smolyak grid) and compute cores of the supercomputer for all cases. For the level-1 Smolyak grid, the number of cores is chosen to be same with the number of points, so each core runs one approximation node corresponding to one optimization problem of the deterministic model (20). For the level-2 Smolyak grid, we see that it will be faster if we use more cores. ${ }^{16}$

From Table 3, we see that the level-2 Chebyshev-Smolyak polynomial approximation obtains about one more digit accuracy than the level-1 ChebyshevSmolyak polynomial approximation for every case. Moreover, $T=50$ does not improve much accuracy in global errors than $T=20$ although it decreases the maximal Euler errors, while $T=50$ is far more time-consuming. With the parallelism, although our examples have far higher numbers of countries than those in Kollmann et al. (2011), we still get the optimal solutions with an acceptable accuracy in minutes, much faster than the other methods listed in Table 3 of Kollmann et al. (2011), except the perturbation methods which will have large errors in the wide domain $[0.7,1.3]^{2 N}$.

\section{Application to a RBC model with a con- straint on investment}

While high dimensionality is an important computational challenge to solving dynamic stochastic problems, low-dimensional problems may have other challenges of their own. For example, if there are occasionally binding constraints for state and/or control variables, then the kinks in the value/policy functions often make the problem difficult to be solved. One advantage of NLCEQ is that it can solve the problems with inequality constraints that occasionally bind. Here we illustrate this by applying NLCEQ to solve a

\footnotetext{
${ }^{16}$ If we can employ a parallel optimization solver for one approximation point so that we can use more cores, then it could be even faster.
} 
RBC model with a constraint on investment.

\subsection{Model Overview}

We use the model in Guerrieri and Iacoviello (2015). That is, we solve the following social planner's problem:

$$
\max _{c} \mathbb{E}\left\{\sum_{t=0}^{\infty} \beta^{t} U\left(c_{t}\right)\right\}
$$

subject to the following constraints

$$
\begin{aligned}
& c_{t}+I_{t}=A_{t} k_{t}^{\alpha}, \\
& k_{t+1}=(1-\delta) k_{t}+I_{t}, \\
& I_{t} \geq \phi I_{s s},
\end{aligned}
$$

for $t \geq 0$, where $c_{t}$ is consumption, $I_{t}$ is investment, $k_{t}$ is capital, and $A_{t}$ is technology following the autoregression process

$$
\ln \left(A_{t+1}\right)=\rho \ln \left(A_{t}\right)+\sigma \epsilon_{t+1},
$$

where $\epsilon_{t}$ is an exogenous innovation with standard normal distribution. We use the parameter values in Guerrieri and Iacoviello (2015), that is, $\beta=$ $0.96, \delta=0.1, \phi=0.975, \alpha=0.33, \rho=0.9, \sigma=0.013, U(c)=\left(c^{1-\gamma}-\right.$ $1) /(1-\gamma)$ with $\gamma=2$. Moreover, $I_{s s}$ is investment in the steady state of the deterministic variant of the model (21) with $A_{t} \equiv 1$. From the first-order conditions for the deterministic variant, we know that the steady state is

$$
k_{s s}=\left(\frac{1}{\alpha}\left(\frac{1}{\beta}-1+\delta\right)\right)^{\frac{1}{\alpha-1}}
$$

and $I_{s s}=\delta k_{s s} \approx 0.3533$. Since the value of $\phi$ is chosen to be close to 1 , the inequality (24) will bind frequently. 


\subsection{Error Measure}

Let $\beta^{t} \lambda_{t}$ denote the Lagrange multiplier of (24) at period $t$. We have the consumption Euler equation and the Kuhn-Tucker condition for (24):

$$
\begin{aligned}
& U^{\prime}\left(c_{t}\right)-\lambda_{t}=\beta \mathbb{E}_{t}\left\{U^{\prime}\left(c_{t+1}\right)\left(1-\delta+\alpha A_{t+1} k_{t+1}^{\alpha-1}\right)-(1-\delta) \lambda_{t+1}\right\} \\
& \lambda_{t}\left(I_{t}-\phi I_{s s}\right)=0
\end{aligned}
$$

Similarly with the examples in the previous section, we use NLCEQ to get the estimate of the optimal consumption function, $C(k, A)$, and the function for the Lagrange multiplier, $\Lambda(k, A)$, on a domain $\left[0.5 k_{s s}, 1.5 k_{s s}\right] \times[0.5,1.5]$. The optimal investment function is $\mathcal{I}(k, A)=A k^{\alpha}-C(k, A)$, and the nextperiod capital is $\mathcal{K}^{+}(k, A)=(1-\delta) k+\mathcal{I}(k, A)$.

Using these approximate functions, for a given $(K, \theta)$, we can compute the following unit-free Euler error:

$$
E_{1}(k, A)=\left|\frac{\beta \mathbb{E}\left\{U^{\prime}\left(c^{+}\right)\left(1-\delta+\alpha A^{+}\left(k^{+}\right)^{\alpha-1}\right)-(1-\delta) \lambda^{+}\right\}+\lambda}{U^{\prime}(c)}-1\right|,
$$

where $A^{+}$is the next-period productivity, $c=C(k, A), \lambda=\Lambda(k, A), k^{+}=$ $\mathcal{K}^{+}(k, A), c^{+}=C\left(k^{+}, A^{+}\right)$, and $\lambda^{+}=\Lambda\left(k^{+}, A^{+}\right)$. We use the 15-point GaussHermite quadrature rule to estimate the integration in (26). Similarly, the unit-free error for the Kuhn-Tucker condition is

$$
E_{2}(k, A)=\left|\lambda\left(\frac{I}{\phi I_{s s}}-1\right)\right|
$$

with $I=\mathcal{I}(k, A)$. The error measure for the investment constraint (24) cannot be omitted, because the true solution of the model without the constraint (24) will also have $E_{1}(k, A)=0$ and $E_{2}(k, A)=0$ with $\lambda=0$, that is, $E_{1}$ and $E_{2}$ are not enough for error measurement. Thus we need to check the 
following unit-free error

$$
E_{3}(k, A)=\max \left(0,1-\frac{I}{\phi I_{s s}}\right) .
$$

We then compute the following global $\mathcal{L}^{\infty}$ and $\mathcal{L}^{1}$ errors on a set of points $(k, A)$, denoted $\mathscr{D}$, to measure the accuracy of our solution:

$$
\begin{gathered}
\mathcal{E}_{\mathcal{L}^{\infty}}=\max _{i=1,2,3}\left\{\max _{(k, A) \in \mathscr{D}} E_{i}(k, A)\right\}, \\
\mathcal{E}_{\mathcal{L}^{1}}=\max _{i=1,2,3}\left\{\frac{1}{|\mathscr{D}|} \sum_{(k, A) \in \mathscr{D}} E_{i}(k, A)\right\},
\end{gathered}
$$

where $|\mathscr{D}|$ is the number of points in the set $\mathscr{D}$. We choose two sets of points, $\mathscr{D}_{1}$ and $\mathscr{D}_{2}$, where $\mathscr{D}_{1}$ is a set of 10,000 randomly and uniformly drawn in $\left[0.7 k_{s s}, 1.3 k_{s s}\right] \times[0.7,1.3],{ }^{17}$ and $\mathscr{D}_{2}$ is a set of 10,000 simulated points in the path of $\left(k_{t}, A_{t}\right)$, where $k_{0}=k_{s s}, A_{0}=1, A_{t+1}$ is simulated based on the stochastic process $(25)$, and $k_{t+1}=\mathcal{K}^{+}\left(k_{t}, A_{t}\right)$ for $t=0, \ldots, 9999$. Thus, $\mathscr{D}_{2}$ represents the ergodic set of $(k, A)$, so the errors on $\mathscr{D}_{2}$ are weighted errors with more weights on the area around the steady state.

\footnotetext{
${ }^{17}$ Guerrieri and Iacoviello (2015) show their results in a much narrower range for $A$, $[0.97,1.025]$. However, our range for $A,[0.7,1.3]$, is reasonable: from $\ln \left(A_{t+1}\right)=\rho \ln \left(A_{t}\right)+$ $\sigma \epsilon_{t+1}$, if $A_{t}$ is inside the following range

$$
\left[\exp \left(\frac{-2 \sigma}{1-\rho}\right), \exp \left(\frac{2 \sigma}{1-\rho}\right)\right]
$$

which is close to $[0.7,1.3]$, then only when $\epsilon_{t+1}$ are always simulated in $[-2,2]$, we can make sure that $A_{t+1}$ is inside the same range. That is, if $A_{t}$ is at one end of the range, then it has about $2.3 \%$ probability that $A_{t+1}$ is outside the range.
} 
Table 4: Errors of the NLCEQ solution with degree- $D$ complete Chebyshev polynomials for the RBC model with a constraint on investment

\begin{tabular}{c|cc|cc|cc|cc}
\hline \multirow{2}{*}{$D$} & \multicolumn{2}{|c|}{ Approx Error for $c$} & \multicolumn{2}{|c|}{ Approx Error for $\lambda$} & \multicolumn{2}{c|}{ Global Error on $\mathscr{D}_{1}$} & \multicolumn{2}{c}{ Global Error on $\mathscr{D}_{2}$} \\
\cline { 2 - 9 } & $\widehat{E}_{\mathcal{L}^{\infty}}$ & $\widehat{E}_{\mathcal{L}^{1}}$ & $\widehat{E}_{\mathcal{L}^{\infty}}$ & $\widehat{E}_{\mathcal{L}^{1}}$ & $\mathcal{E}_{\mathcal{L}^{\infty}}$ & $\mathcal{E}_{\mathcal{L}^{1}}$ & $\mathcal{E}_{\mathcal{L}^{\infty}}$ & $\mathcal{E}_{\mathcal{L}^{1}}$ \\
\hline 10 & $5.2(-3)$ & $1.7(-3)$ & $2.5(-2)$ & $5.4(-3)$ & $2.6(-2)$ & $3.1(-3)$ & $2.1(-2)$ & $2.5(-3)$ \\
20 & $2.6(-3)$ & $5.0(-4)$ & $1.5(-2)$ & $1.4(-3)$ & $1.3(-2)$ & $7.9(-4)$ & $5.3(-3)$ & $6.9(-4)$ \\
50 & $1.6(-3)$ & $8.5(-5)$ & $9.8(-3)$ & $2.7(-4)$ & $4.5(-3)$ & $1.4(-4)$ & $4.9(-4)$ & $9.7(-5)$ \\
100 & $8.2(-4)$ & $2.1(-5)$ & $2.2(-3)$ & $6.9(-5)$ & $2.0(-3)$ & $1.2(-4)$ & $1.9(-3)$ & $1.8(-4)$ \\
\hline
\end{tabular}

Note: $\zeta(-j)$ means $\zeta \times 10^{-j}$.

\subsection{Numerical Results}

In the transformation step of the NLCEQ method, we choose $T=100$ and the problem becomes

$$
\widetilde{V}\left(k_{0}, A_{0}\right)=\max _{c} \sum_{t=0}^{T-1} \beta^{t} U\left(c_{t}\right)+\beta^{T} \widetilde{V}_{T}\left(k_{T}, A_{T}\right),
$$

subject to (22)-(24) with a deterministic process of $A_{t}: \ln \left(A_{t+1}\right)=\rho \ln \left(A_{t}\right)$. The terminal value function $\widetilde{V}_{T}(k, A)$ is given as $U\left(0.7 A k^{\alpha}\right) /(1-\beta)$. In the approximation step of NLCEQ, we use the tensor grid of Chebyshev nodes $(D+1$ nodes in each dimension) and degree- $D$ complete Chebyshev polynomials.

Table 4 reports approximation errors and global errors of the solution of NLCEQ over two sets of points, $\mathscr{D}_{1}$ and $\mathscr{D}_{2}$, for various degrees $D$. We see that higher degree approximation achieves higher accuracy, and the weighted errors on $\mathscr{D}_{2}$ are a bit smaller than those on $\mathscr{D}_{1}$. Because of the kinks caused by the frequently binding constraint on investment, a polynomial approximation is not very good at approximating functions with kinks until a high degree approximation (this is reflected by the approximation errors of Lagrange multiplier $\lambda$ in the table, moreover most of global errors in the table come from the investment constraint error $E_{3}$ because of the kinks on the investment function), so NLCEQ achieves an accuracy with $O\left(10^{-3}\right)$ in $\mathcal{L}^{\infty}$ 
or $O\left(10^{-4}\right)$ in $\mathcal{L}^{1}$ until the degree-50 approximation. ${ }^{18}$

However, the order-1 perturbation (log-linearization) method has an $\mathcal{L}^{\infty}$ global error up to 0.73 and an $\mathcal{L}^{1}$ global error up to 0.17 on the domain $\left[0.7 k_{s s}, 1.3 k_{s s}\right] \times[0.7,1.3]$, although its $\mathcal{L}^{\infty}$ error is 0.02 and $\mathcal{L}^{1}$ error is 0.003 for the model without the investment constraint (24). The order-2 perturbation method does not improve the accuracy as its $\mathcal{L}^{\infty}$ error is 0.8 and $\mathcal{L}^{1}$ error is 0.18., although it increases about two order accuracy for the model without the investment constraint (24). Therefore, this shows that NLCEQ is much more accurate, about two or three orders of magnitude higher, than the order- 1 and order- 2 perturbation methods for this problem with the occasionally binding constraint.

The comparison between NLCEQ and log-linearization is also shown in Figure 1, which shows the global errors of their solutions when $A=0.7,1$, and 1.3. The NLCEQ solution is the one with degree-100 complete Chebyshev polynomial approximation. Figure 1 shows clearly that NLCEQ is much more accurate than log-linearization globally, particularly when the state is not close to the steady state.

We now try piecewise bilinear interpolation as the approximation method, because piecewise bilinear interpolation can deal with the kinks better than polynomials. For the approximation nodes, we choose the tensor grid of $n$ equally spaced capital in $\left[0.5 k_{s s}, 1.5 k_{s s}\right]$ and $n$ equally spaced productivity in $[0.5,1.5]$. Table 5 lists approximation errors and global errors from NLCEQ with piecewise bilinear interpolation, and we found that the piecewise bilinear interpolation has smaller, about one order of magnitude, errors than the complete Chebyshev polynomials when they use the same number of approximation nodes.

Table 6 shows global errors for various standard deviation $\sigma$ (approximation errors are independent on $\sigma$ ). We see that a smaller $\sigma$ has smaller

\footnotetext{
${ }^{18}$ We also tried the case with $\phi=0$, and found that its NLCEQ solution has a bit smaller errors than those in Table 4.
} 
Figure 1: Errors of the solutions from NLCEQ or log-linearization for the $\mathrm{RBC}$ model with a constraint on investment

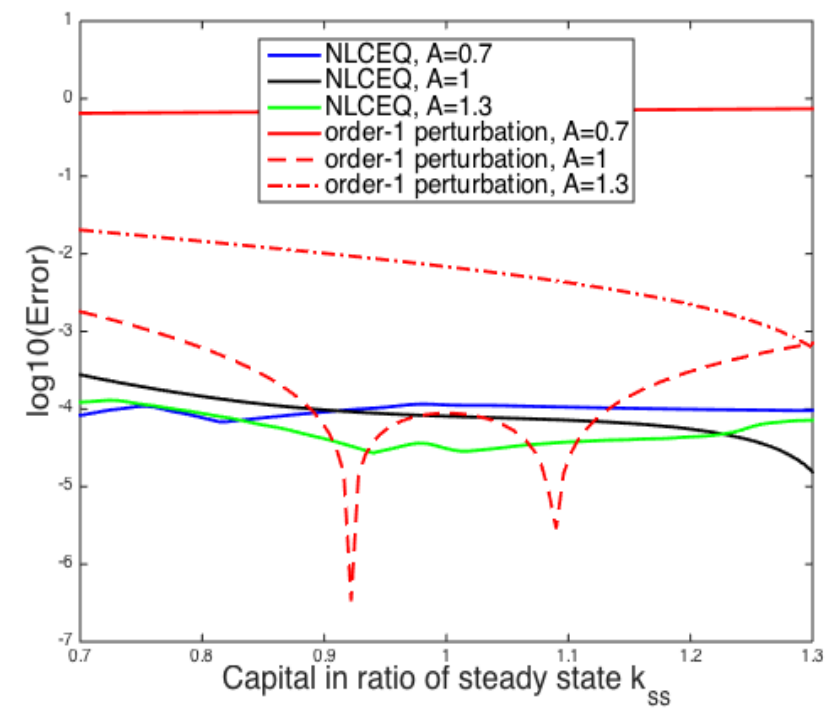

Table 5: Errors of the NLCEQ solution with piecewise bilinear interpolation for the RBC model with a constraint on investment

\begin{tabular}{c|cc|cc|cc|cc}
\hline \multirow{2}{*}{$n$} & \multicolumn{2}{|c|}{ Approx } & Error for $c$ & \multicolumn{2}{|c|}{ Approx Error for $\lambda$} & \multicolumn{2}{c|}{ Global Error on $\mathscr{D}_{1}$} & \multicolumn{2}{c}{ Global Error on $\mathscr{D}_{2}$} \\
\cline { 2 - 9 } & $\widehat{E}_{\mathcal{L}^{\infty}}$ & $\widehat{E}_{\mathcal{L}^{1}}$ & $\widehat{E}_{\mathcal{L}^{\infty}}$ & $\widehat{E}_{\mathcal{L}^{1}}$ & $\mathcal{E}_{\mathcal{L}^{\infty}}$ & $\mathcal{E}_{\mathcal{L}^{1}}$ & $\mathcal{E}_{\mathcal{L}^{\infty}}$ & $\mathcal{E}_{\mathcal{L}^{1}}$ \\
\hline 21 & $3.7(-3)$ & $1.1(-4)$ & $4.0(-2)$ & $3.6(-3)$ & $5.8(-3)$ & $7.6(-4)$ & $1.7(-3)$ & $3.1(-4)$ \\
51 & $1.9(-3)$ & $2.6(-5)$ & $7.3(-3)$ & $5.9(-4)$ & $8.7(-4)$ & $1.7(-4)$ & $4.5(-4)$ & $1.1(-4)$ \\
101 & $7.5(-4)$ & $4.1(-6)$ & $4.7(-3)$ & $1.4(-4)$ & $3.6(-4)$ & $1.1(-4)$ & $2.5(-4)$ & $9.8(-5)$ \\
\hline
\end{tabular}

Note: $\zeta(-j)$ means $\zeta \times 10^{-j}$. 
Table 6: Errors of the NLCEQ solution with piecewise bilinear interpolation for the RBC model with a constraint on investment and various standard deviations

Note: $\zeta(-j)$ means $\zeta \times 10^{-j}$.

\begin{tabular}{c|c|cc|cc}
\hline$\sigma$ & $n$ & \multicolumn{2}{|c|}{ Global Error on $\mathscr{D}_{1}$} & \multicolumn{2}{c}{ Global } \\
\cline { 3 - 6 } & & $\mathcal{E}_{\mathcal{L}^{\infty}}$ & $\mathcal{E}_{\mathcal{L}^{1}}$ & $\mathcal{E}_{\mathcal{L}^{\infty}}$ & $\mathcal{E}_{\mathcal{L}^{1}}$ \\
\hline 0.001 & 21 & $7.5(-3)$ & $8.4(-4)$ & $2.5(-4)$ & $3.5(-5)$ \\
& 51 & $9.3(-4)$ & $1.2(-4)$ & $4.5(-5)$ & $8.2(-6)$ \\
& 101 & $2.9(-4)$ & $3.1(-5)$ & $3.0(-6)$ & $6.8(-7)$ \\
\hline 0.02 & 21 & $5.6(-3)$ & $7.9(-4)$ & $2.0(-3)$ & $4.6(-4)$ \\
& 51 & $1.3(-3)$ & $2.8(-4)$ & $7.9(-4)$ & $2.4(-4)$ \\
& 101 & $6.6(-4)$ & $2.4(-4)$ & $4.2(-4)$ & $2.3(-4)$ \\
\hline 0.05 & 21 & $8.1(-3)$ & $1.8(-3)$ & $9.4(-3)$ & $1.5(-3)$ \\
& 51 & $2.9(-3)$ & $1.4(-3)$ & $5.9(-3)$ & $1.4(-3)$ \\
& 101 & $2.8(-3)$ & $1.4(-3)$ & $4.0(-3)$ & $1.3(-3)$ \\
\hline
\end{tabular}

errors and it has about four-digit accuracy for the smallest $\sigma=0.001$. When $\sigma=0.05$, the errors are up to $O\left(10^{-3}\right)$ and there are almost no improvement by increasing $n$ from 51 to 101. Moreover, when $\sigma$ is up to 0.05, the global errors on the ergodic set $\mathscr{D}_{2}$ are bigger than those on $\mathscr{D}_{1}$, because the domain containing $\mathscr{D}_{1},\left[0.7 k_{s s}, 1.3 k_{s s}\right] \times[0.7,1.3]$, is not large enough to contain $\mathscr{D}_{2}$ for large $\sigma$.

However, the errors for large $\sigma$ can be decreased by changing the deterministic transition law of $A_{t}$ to $\ln \left(A_{t+1}\right)=\rho \ln \left(A_{t}\right)-0.5 \sigma^{2}$. Table 7 shows errors for $\sigma=0.05$ using the new deterministic transition law of $A_{t}$ and piecewise bilinear interpolation. We see that the errors are smaller than those in 6 from $\ln \left(A_{t+1}\right)=\rho \ln \left(A_{t}\right)$. Moreover, a larger $n$ clearly improves the accuracy of the solution.

Since global errors cannot represent true errors compared with the true solution, we implement shape-preserving value function iteration with rational spline interpolation (Cai and Judd 2012) to derive the "true" solution and then check the "true" errors. We follow Tauchen (1986) to approximate the process of $\ln \left(A_{t}\right)$ with a Markov chain of 101 equally spaced values in 
Table 7: Errors of the NLCEQ solution using $\ln \left(A_{t+1}\right)=\rho \ln \left(A_{t}\right)-0.5 \sigma^{2}$

Note: $\zeta(-j)$ means $\zeta \times 10^{-j}$.

\begin{tabular}{c|c|cc|cc}
\hline$\sigma$ & \multirow{2}{*}{$n$} & \multicolumn{2}{|c|}{ Global Error on $\mathscr{D}_{1}$} & \multicolumn{2}{c}{ Global Error on $\mathscr{D}_{2}$} \\
\cline { 3 - 6 } & & $\mathcal{E}_{\mathcal{L}^{\infty}}$ & $\mathcal{E}_{\mathcal{L}^{1}}$ & $\mathcal{E}_{\mathcal{L}^{\infty}}$ & $\mathcal{E}_{\mathcal{L}^{1}}$ \\
\hline 0.05 & 21 & $5.8(-3)$ & $9.5(-4)$ & $7.5(-3)$ & $7.8(-4)$ \\
& 51 & $1.8(-3)$ & $5.1(-4)$ & $4.9(-3)$ & $4.6(-4)$ \\
& 101 & $1.7(-3)$ & $4.7(-4)$ & $3.5(-3)$ & $3.9(-4)$ \\
\hline
\end{tabular}

Table 8: "True" relative errors of the NLCEQ solution for the RBC model with a constraint on investment

\begin{tabular}{c|cc|cc}
\hline$n$ & \multicolumn{2}{|c|}{ Piecewise Bilinear Interp. } & \multicolumn{2}{c}{ Complete Chebyshev Poly. } \\
\cline { 2 - 5 } & error in $\mathcal{L}^{\infty}$ & error in $\mathcal{L}^{1}$ & error in $\mathcal{L}^{\infty}$ & error in $\mathcal{L}^{1}$ \\
\hline 21 & $6.0(-3)$ & $3.2(-4)$ & $5.2(-3)$ & $1.3(-3)$ \\
51 & $3.2(-3)$ & $1.5(-4)$ & $2.7(-3)$ & $2.5(-4)$ \\
101 & $4.2(-4)$ & $1.1(-5)$ & $1.3(-3)$ & $1.5(-4)$ \\
\hline
\end{tabular}

Note: $\zeta(-j)$ means $\zeta \times 10^{-j}$.

$[0.5,1.5]$, and use 101 equally spaced nodes for capital in $\left[0.5 k_{s s}, 1.5 k_{s s}\right]$ as the approximation nodes for the rational spline interpolation for each discrete value of the Markov process $\ln \left(A_{t}\right)$. The value function iteration stops while the relative change of two consecutive value functions is less than $10^{-6}$. With these converged "true" solution, Table 8 reports "true" relative errors for consumption function in the domain of $k$ and $A,\left[0.7 k_{s s}, 1.3 k_{s s}\right] \times[0.7,1.3]$, from NLCEQ with degree- $(n-1)$ complete Chebyshev polynomials or piecewise bilinear interpolation with $n \times n$ approximation nodes. We see that these errors are close to those global errors in Table 4 or Table 6 . We also see that the "true" relative errors from piecewise bilinear interpolation are smaller than those from complete Chebyshev polynomials when $n=101$.

Figure 2 shows the optimal investment policy functions from NLCEQ with piecewise bilinear interpolation $(n=101)$. We see that when technology $A_{t}>1$ and capital $k_{t}>0.7 k_{s s}$, the investment is always bigger than its lower bound. But if $A_{t}$ is small then the investment is binding at the lower bound. 
Figure 2: Investment policy function for the RBC model with a constraint on investment
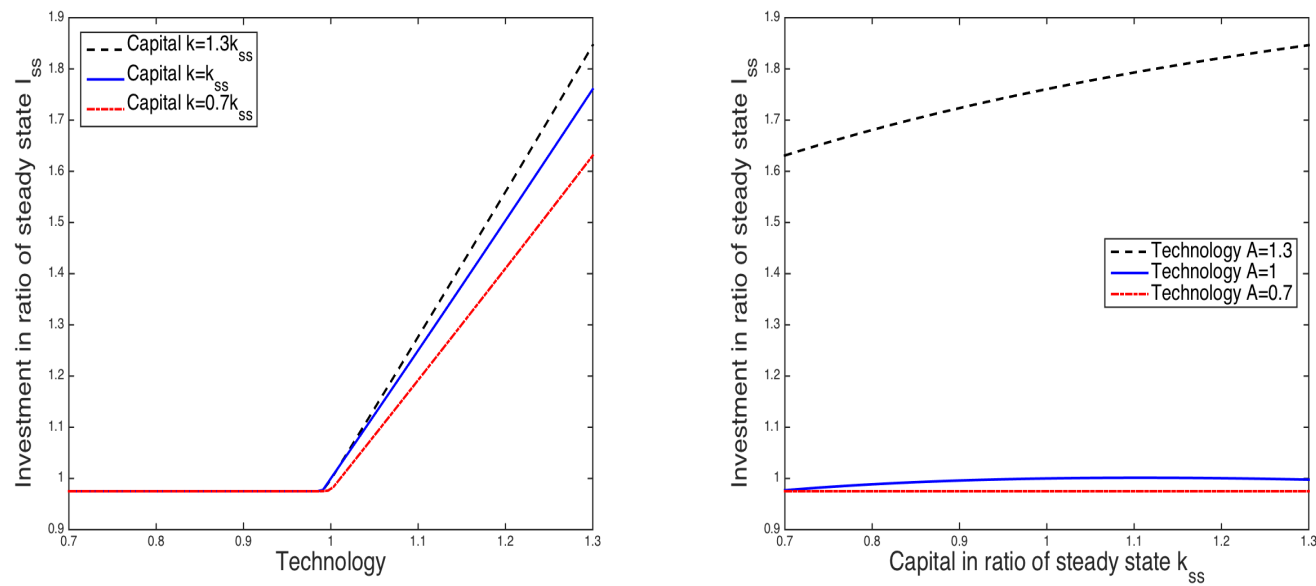

\section{Application to a dynamic stochastic model of food and clean energy}

In previous section we solved problems with occasionally binding constraints and their steady state in the center of their state space. However, in some problems like the one shown below, the steady state is on the boundary of the feasible space of states, and is approached from only one side. Moreover, we know that usually the solution at the initial states is the most important, but the initial states could be far away from the steady state. For these problems, log-linearization is not reliable because it can only give good solutions around the steady state. This section applies NLCEQ to solve such a low-dimensional problem, where there are no reachable steady states, its initial states stay in a corner region, and there are inequality constraints that occasionally bind. 


\section{$5.1 \quad$ Model Setup}

In this example we apply NLCEQ method to solve a stochastic version of a dynamic model of food and clean energy introduced by Chakravorty et al. (2008). This stylized model serves as a vehicle for developing and solving more complex models aimed at understanding complicated real world economic problems related to biofuels and global land use. ${ }^{19}$

We assume a single-country economy with two primary factors, land and fossil fuels (e.g., oil). The economy has a fixed endowment of land, $L$, which can be used to produce food or biofuels. Let $L_{t, f}$ and $L_{t, b}$ be the amounts of land dedicated to produce food and biofuels crops at time $t$, respectively. The residual land, $L-L_{t, f}-L_{t, b}$, is unused. Total land constraint is:

$$
L_{t, f}+L_{t, b} \leq L
$$

The economy has also some stock of extractable fossil fuel resource (e.g., oil), $S$, with the initial stock $S_{0}$. At period $t$ the economy extracts $s_{t}$ units of fossil fuel, so we have:

$$
S_{t+1}=S_{t}-s_{t}
$$

Production of food employs only land resource. The production function for food crops is linear in the amount of land used. There is one stochastic tipping event: once it happens, it adversely affects the production of food crops at a level $\bar{J}<1$, and this damage is irreversible for any later periods. Let the food production per unit of land be $\theta_{t, f}$ before the tipping event happens (we assume $\theta_{t, f}=1$ for simplicity). Thus, the production function

19 For direct model extension see Chakravorty et al. (2012). Steinbuks and Hertel (2014) present a closely related computable partial equilibrium model of land use at the global scale, which incorporates additional sectors and non-homothetic preferences. While all these works assume perfect foresight, Cai el al. (2014) incorporate uncertainty in global land use decision models. 
for food crops is

$$
y_{t, f} \equiv\left(1-J_{t}\right) \theta_{t, f} L_{t, f}
$$

where $J_{t}$ denotes the stochastic damage level: $J_{t}=0$ if the tipping event has not happened before time $t$, otherwise $J_{t}=\bar{J}$. We assume that the stochastic process $J_{t}$ is a Markov chain with the transition probability matrix

$$
P=\left(\begin{array}{cc}
1-p_{21} & 0 \\
p_{21} & 1
\end{array}\right)
$$

where $p_{21}$ is the probability that the tipping event happens in one year, and the $(2,2)$ element of $P$ is 1 because of the irreversibility of the tipping damage. ${ }^{20}$

Production of energy employs both fossil fuels and biofuels, and it is a constant elasticity of substitution (CES) function: ${ }^{21}$

$$
y_{t, e} \equiv A\left[\alpha\left(\theta_{t, b} L_{t, b}\right)^{\lambda}+(1-\alpha)\left(s_{t}\right)^{\lambda}\right]^{\frac{1}{\lambda}},
$$

where $A$ is the technology parameter of energy production, $\theta_{t, b}$ is the return of biofuels crops per unit of land (we assume $\theta_{t, b}=1$ for simplicity), $\alpha$ is the cost share of biofuels' feedstocks and $\lambda$ is the CES function parameter proportional to the elasticity of substitution of oil for biofuels.

Let $M$ be the mass of pollution (e.g., carbon concentration), with the

${ }^{20}$ Cai et al. (2015a) assume a more general tipping process with irreversible damages on production. We use this simpler version for illustrating the application of NLCEQ without loss of generality.

${ }^{21}$ Chakravorty et al. (2008) assume that fossil fuels and biofuels are perfect substitutes. Our modification of the original model makes it more realistic (as biofuels substitute imperfectly for petroleum in final liquid fuel demand), and avoids numerical problems caused by bang-bang solutions of Chakravorty et al. (2008). 
initial stock $M_{0}$. The law of accumulation of pollution is:

$$
M_{t+1}=\mu s_{t}+(1-\delta) M_{t}
$$

where $\mu$ is the amount of pollution produced from combustion of one unit of fossil fuel (relative to biofuels), and $\delta$ is the natural rate of pollution absorption by the earth atmosphere and oceans.

The non-land production costs of food and biofuels are linear:

$$
c_{t, j} \equiv \psi_{j} L_{t, j}
$$

where $\psi_{j}$ is the food or biofuels cost per unit of land, for $j \in\{f, b\}$. The fossil fuel extraction cost is:

$$
c_{t, e} \equiv \psi_{1 e} s_{t}\left(S_{t}\right)^{-\psi_{2 e}},
$$

where $\psi_{1 e}$ and $\psi_{2 e}$ are two positive parameters, so that oil extraction cost increases with depletion of the oil stock $S_{t}{ }^{22}$ Following Cai et al. (2014) we assume that at each time $t$ there is an exogenous endowment of other primary resources (e.g., labor, physical and human capital, and materials), $\Pi_{t}$. A part of this endowment is used in food and energy sectors for oil extraction and refining, and production of food and biofuels. The remaining amount of other primary resources is converted to other goods, which are consumed in final demand. The production of other goods is linear in the remaining amount of other primary resources with transformation coefficient $\theta_{t, o}\left(\right.$ for simplicity we assume $\left.\theta_{t, o}=1\right)$ :

$$
y_{t, o} \equiv \theta_{t, o}\left(\Pi_{t}-c_{t, f}-c_{t, b}-c_{t, e}\right) .
$$

\footnotetext{
${ }^{22}$ This is another modification of Chakravorty et al. (2008), who assume linear extraction costs for simplicity. Our cost specification is more commonly used in the environmental economics literature (see e.g., Nordhaus and Boyer 2003).
} 
We assume a utility function, which is additively separable in food, energy, and other goods (positively), as well as pollution stock (negatively):

$$
u\left(y_{t, f}, y_{t, e}, y_{t, o}, M_{t}\right)=\frac{\left(y_{t, f}\right)^{1-\frac{1}{\gamma_{f}}}}{1-\frac{1}{\gamma_{f}}}+B_{e} \frac{\left(y_{t, e}\right)^{1-\frac{1}{\gamma_{e}}}}{1-\frac{1}{\gamma_{e}}}+B_{o} \frac{\left(y_{t, o}\right)^{1-\frac{1}{\gamma_{o}}}}{1-\frac{1}{\gamma_{o}}}-B_{M} M_{t}^{\eta}
$$

where $\gamma_{f}, \gamma_{e}, \gamma_{o}, B_{e}, B_{o}, B_{M}$, and $\eta$ are positive parameters. ${ }^{23}$ In addition, following Chakravorty et al. (2008) we assume that pollution stock is capped at certain threshold by an international agreement, which is not necessarily consistent with unconstrained country pollution optimum. That is, we assume that $M_{t} \leq \bar{M}$ for all $t$ with a given upper bound $\bar{M}$.

The objective of the social planner is to maximize the expected sum of the discounted utility with a discount factor $\beta$. That is, the social planner's problem is

$$
V\left(S_{0}, M_{0}, J_{0}\right)=\max _{L_{t, f}, L_{t, b}, s_{t} \geq 0} \mathbb{E}\left\{\sum_{t=0}^{\infty} \beta^{t}\left[u\left(y_{t, f}, y_{t, e}, y_{t, o}, M_{t}\right)\right]\right\}
$$

subject to (28), (29), (33), and $M_{t} \leq \bar{M}$, with three nonnegative control variables at each time $t$ : land dedicated to food crops $L_{t, f}$, land dedicated to biofuels $L_{t, b}$, and extracted fossil fuels $s_{t}$.

There are two endogenous state variables: fossil fuel stock, $S_{t}$, governed by equation (29), and accumulated pollution, $M_{t}$, governed by equation (33), and one exogenous discrete state variable: damage level, $J_{t}$. Note that the steady endogenous states imply zero extraction of fossil fuels and zero pollution from (29) and (33), i.e., $s_{t}=0$ and $M_{t}=0$. This means that the steady state will not be reached in the optimal path in a finite horizon as $M_{t}$ is always bigger than 0 in our model (although $M_{t}$ converges to 0 as $t$ goes

\footnotetext{
${ }^{23}$ This formulation is different from Chakravorty et. al. (2008), who do not incorporate disutility from pollution in the utility function, but it is broadly consistent with the environmental economics and growth literature, see e.g., Andreoni and Levinson (2001), and Xepapadeas (2005).
} 
to infinite).

We set the total amount of land, $L$, equal to 1 . We also assume that the tipping event has not happened at the initial time (i.e., $J_{0}=0$ ). In our example, we let $\bar{J}=0.1, p_{21}=0.0034, \beta=0.95, \alpha=0.5, \lambda=0.5, \delta=0.001$, $\mu=0.25, \gamma_{f}=\gamma_{e}=\gamma_{o}=0.5, B_{e}=B_{o}=0.5, B_{M}=1, \eta=4, \Pi_{t} \equiv 1, A=1$, $\psi_{1 e}=0.4, \psi_{2 e}=1, \psi_{f}=0.3, \psi_{b}=0.5$, and $\bar{M}=1.06$.

\subsection{Numerical Results}

Since $S_{t}$ is always non-increasing over time and $M_{t}$ has an upper bound $\bar{M}=1.06$, we set the approximation domain for the value/policy functions as $S_{0} \in[0.01,1]$ and $M_{0} \in[1,1.06]$, for each $J_{0} \in\{0, \bar{J}\}$. We set the length of time path equal to $T=200$ periods for the dynamic model of food and clean energy in the deterministic model transformed from (38) in the transformation step of NLCEQ (Algorithm 1). In the transformation step, if $J_{0}=0$, then we change $J_{t}$ to its unconditional expectation at time $t, p_{t, 1,2} \bar{J}$, where $p_{t, 1,2}=1-\left(1-p_{21}\right)^{t}$ (the second element of the vector $P^{t}(1,0)^{\top}$ with $P$ given by (31)) is the probability that the tipping event happens at a time not later than $t$; if $J_{0}=\bar{J}$, then it has been a deterministic model as $J_{t}$ will always be $\bar{J}$ because of the irreversibility of the tipping damage. We assume the terminal value function to be $u\left(y_{T, f}, y_{T, e}, y_{T, o}, M_{T}\right) /(1-\beta)$ where $\left(y_{T, f}, y_{T, e}, y_{T, o}\right)$ are given by (30), (32), and (36) with terminal extraction $s_{T}=0.01 X_{T}$.

For this specific problem, we can compute the true value/policy functions of the model (38) in order to measure the accuracy of solutions from NLCEQ, so we do not need to test the accuracy using its Euler equations errors like what we did for the RBC model (11). When $J_{0}=0$, the problem can be solved as an optimal control problem by a large-scale optimization solver in 
Table 9: Errors of Policy Functions from NLCEQ for the Model of Food and Clean Energy

\begin{tabular}{c|cc|cc}
\hline$D$ & \multicolumn{2}{|c|}{ Error for $L_{f}$} & \multicolumn{2}{c}{ Error for $s$} \\
& $\mathcal{L}^{\infty}$ & $\mathcal{L}^{1}$ & $\mathcal{L}^{\infty}$ & $\mathcal{L}^{1}$ \\
\hline 4 & $9.4(-3)$ & $1.2(-3)$ & $1.8(-3)$ & $2.8(-4)$ \\
6 & $3.7(-3)$ & $5.8(-4)$ & $1.1(-3)$ & $1.6(-4)$ \\
8 & $2.2(-3)$ & $3.0(-4)$ & $7.8(-4)$ & $9.5(-5)$ \\
10 & $2.1(-3)$ & $1.8(-4)$ & $6.0(-4)$ & $6.1(-5)$ \\
20 & $8.4(-4)$ & $4.8(-5)$ & $2.6(-4)$ & $1.9(-5)$ \\
\hline Note: $\zeta(-j)$ means $\zeta \times 10^{-j}$. &
\end{tabular}

the following form:

$$
V_{J_{0}=0}\left(S_{0}, M_{0}\right)=\max _{L_{t, f}, L_{t, b}, s_{t} \geq 0}\left\{\sum_{t=0}^{T} \beta^{t}\left[\sum_{j=1}^{2} p_{t, 1, j} u\left(y_{t, f, j}, y_{t, e}, y_{t, o}, M_{t}\right)\right]\right\}
$$

subject to (28), (29), (33), and $M_{t} \leq \bar{M}$, where $p_{t, 1,1}=1-p_{t, 1,2}, y_{t, f, 1}=$ $\theta_{t, f} L_{t, f}$, and $y_{t, f, 2}=(1-\bar{J}) \theta_{t, f} L_{t, f}$. When $J_{0}=\bar{J}$, the problem is deterministic:

$$
V_{J_{0}=\bar{J}}\left(S_{0}, M_{0}\right)=\max _{L_{t, f}, L_{t, b}, s_{t} \geq 0}\left\{\sum_{t=0}^{T} \beta^{t}\left[u\left(y_{t, f, 2}, y_{t, e}, y_{t, o}, M_{t}\right)\right]\right\}
$$

subject to (28), (29), (33), and $M_{t} \leq \bar{M}$.

We use the initial-time solutions for the control variables at approximation nodes of $S_{0}$ and $M_{0}$ to construct the optimal policy functions for each $J_{0} \in\{0, \bar{J}\}$. n the NLCEQ method, we use the tensor grid of Chebyshev nodes ( $D+1$ nodes in each dimension) over the domain of the continuous state variables, $[0.01,1] \times[1,1.06]$, in the optimization step. We apply the degree- $D$ complete Chebyshev polynomials in the approximation step, for each $J_{0} \in\{0, \bar{J}\}$.

Table 9 reports absolute errors ${ }^{24}$ of the policy functions computed by

\footnotetext{
${ }^{24}$ We do not use relative errors because the solution of the fossil fuel extraction $s$ could
} 
Figure 3: Pre-tipping State Paths for the Model of Food and Clean Energy
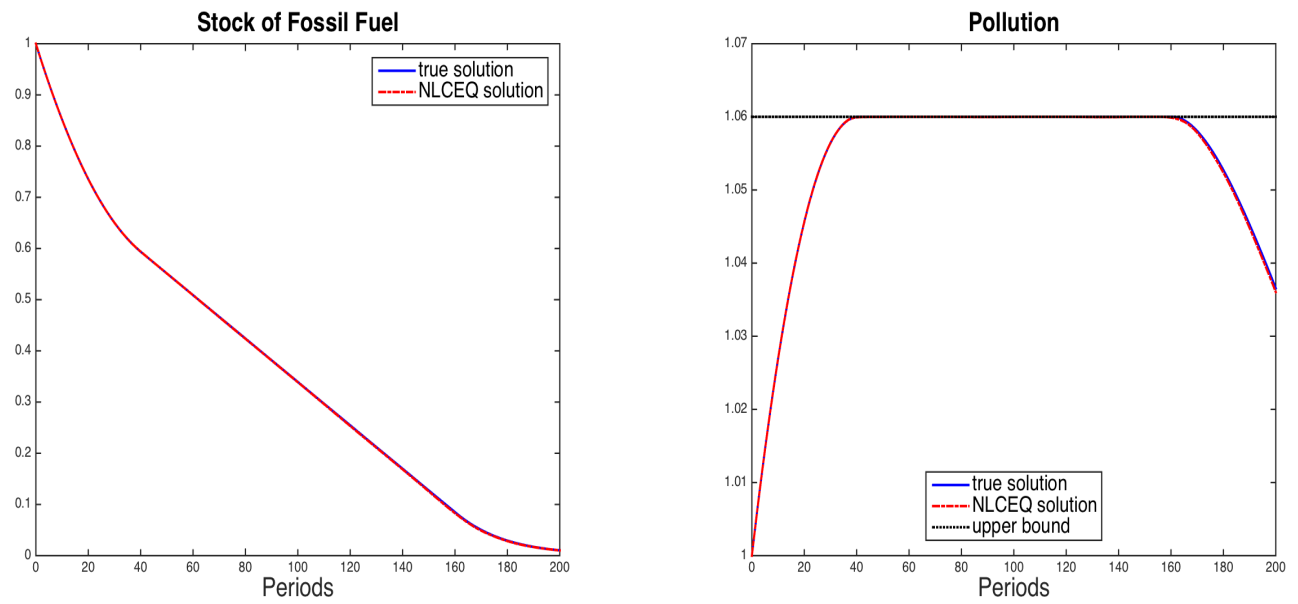

NLCEQ over the approximation domain and all discrete state values for various degrees $D$. We see that NLCEQ gives $O\left(10^{-4}\right)$ accuracy for the policy functions, after we use the degree-20 polynomial approximation. Figure 3 shows the pre-tipping paths of stock of fossil fuel $X_{t}$ and pollution $M_{t}$, and we see that our NLCEQ solutions are very close to true solutions, and $M_{t}$ hits its upper bound after 40 periods and sticks on the bound for more than 100 periods.

\section{Application to a New Keynesian Model with Zero Lower Bound}

In this section, we apply NLCEQ for competitive equilibrium (Algorithm 2) to solving a New Keynesian model with zero lower bound (ZLB). We use the New Keynesian model in Guerrieri and Iacoviello (2015), a variant of the new Keynesian model with ZLB that is used in Fernández-Villaverde et al. (2015) and Maliar and Maliar (2015). The values of parameters are also be nearly 0 . 
chosen from Guerrieri and Iacoviello (2015).

\subsection{Model Overview}

The model consists of a representative household, a government, a final-good firm, and intermediate firms. At each time $t$ the government issues bonds that expire at $t+1$ and the nominal interest rate for the bonds is $r_{t}$ (the time unit is a quarter). A representative household consumes $c_{t}$ with a price $p_{t}$ from the final-good firm, buys newly issued bonds with a total face value $b_{t}$ from the government, sells the expired bonds $b_{t-1}$, earns wages from labor supply $\ell_{t}$ with a wage rate $w_{t}$, and receives a lump-sum transfer $T_{t}$ from the government and profit $\Pi_{t}$ from all firms. The budget constraint is as follows:

$$
p_{t} c_{t}+\frac{b_{t}}{1+r_{t}}=w_{t} \ell_{t}+b_{t-1}+T_{t}+\Pi_{t}
$$

The representative household chooses consumption $c_{t}$, labor supply $\ell_{t}$, and government bonds $b_{t}$ to maximize

$$
\max _{c_{t}, \ell_{t}, b_{t}} \mathbb{E}\left\{\sum_{t=0}^{\infty}\left(\prod_{i=0}^{t} \beta_{i}\right) U\left(c_{t}, \ell_{t}\right)\right\}
$$

subject to the budget constraint (41), where

$$
U(c, \ell)=\ln (c)-\frac{\ell^{1+\eta}}{1+\eta}
$$

with $\eta=1$. The discount factor $\beta_{t}$ is a stochastic process following

$$
\ln \left(\beta_{t+1}\right)=(1-\rho) \ln \left(\beta^{*}\right)+\rho \ln \left(\beta_{t}\right)+\sigma \epsilon_{t+1}
$$

where $\epsilon_{t} \sim$ i.i.d.N $\mathcal{N}(0,1), \beta^{*}=0.994, \rho=0.8$, and $\sigma=0.005$. The first-order 
conditions of the household problem imply

$$
1=\mathbb{E}_{t}\left\{\beta_{t+1} \frac{1+r_{t}}{\pi_{t+1}} \frac{c_{t}}{c_{t+1}}\right\}
$$

and

$$
w_{t}=p_{t} c_{t} \ell_{t}^{\eta}
$$

where $\pi_{t} \equiv p_{t} / p_{t-1}$ is the gross inflation rate.

The final-good firm purchases intermediate goods from intermediate firms to produce a final good $y_{t}$ and sell it at a price $p_{t}$. The intermediate firms are assumed to have Calvo-type prices: a fraction $1-\theta$ of the firms have optimal prices and the remaining fraction $\theta$ of the firms keep the same price as in the previous period. Here the Calvo parameter $\theta$ is set as 0.9. In Appendix B we describe the detailed model specification for the final- and intermediategoods firms and derive the following equilibrium conditions:

$$
\begin{aligned}
1 & =\frac{1}{\chi_{t, 1}}\left(y_{t} \ell_{t}^{\eta}+\theta \mathbb{E}_{t}\left\{\beta_{t+1} \pi_{t+1}^{\alpha} \chi_{t+1,1}\right\}\right) \\
1 & =\frac{1}{\chi_{t, 2}}\left(\frac{y_{t}}{c_{t}}+\theta \mathbb{E}_{t}\left\{\beta_{t+1} \pi_{t+1}^{\alpha-1} \chi_{t+1,2}\right\}\right) \\
q_{t} & =\frac{\alpha \chi_{t, 1}}{(\alpha-1) \chi_{t, 2}}=\left(\frac{1-\theta \pi_{t}^{\alpha-1}}{1-\theta}\right)^{\frac{1}{1-\alpha}} \\
v_{t+1} & =\frac{\ell_{t}}{y_{t}}=(1-\theta) q_{t}^{-\alpha}+\theta \pi_{t}^{\alpha} v_{t}
\end{aligned}
$$

where $\alpha=6$, for any time $t \geq 0$.

Let $\pi^{*}, r^{*}$, and $y^{*}$ be the steady-state gross level of inflation, the steadystate nominal interest rate, and the steady-state output, respectively. Let the government spending $g_{t}$ be always equal to $s_{g} y_{t}$ with $s_{g}=0.2$. From the market clearing condition $y_{t}=c_{t}+g_{t}$, we have

$$
c_{t}=\left(1-s_{g}\right) y_{t}
$$


Following the Taylor rule (Taylor 1993), we have the nominal interest rate as

$$
r_{t}=\max \left(z_{t}, 0\right)
$$

with

$$
z_{t}=\left(1+r^{*}\right)\left(\frac{\pi_{t}}{\pi^{*}}\right)^{\phi_{\pi}}\left(\frac{y_{t}}{y^{*}}\right)^{\phi_{y}}-1
$$

where we choose $\phi_{\pi}=2.5, \phi_{y}=0.25$, and $\pi^{*}=1.005$. We have $r^{*}=\pi^{*} / \beta^{*}-1$ from (44), and the formula for $y^{*}$ is given in Appendix C. Equation (51) implies that the actual policy rate $r_{t}$ must be nonnegative, and this zero lower bound will be binding when the notional policy rate $z_{t}$ is smaller than 0 .

We now have one endogenous state variable $v_{t}$ and one exogenous state variable $\beta_{t}$, the system of equilibrium equations (44) and (46)-(52), and the exogenous process (43). We apply NLCEQ (Algorithm 2) to compute the policy functions for $\left(c_{t}, \chi_{t, 1}, \chi_{t, 2}, \pi_{t}, q_{t}, v_{t}, \ell_{t}, y_{t}, r_{t}, z_{t}\right)$, and with the NLCEQ solution it follows that the consumption price is computed by $p_{t}=\pi_{t} p_{t-1}$ and then the wage is computed by (45).

\subsection{Numerical Results}

In NLCEQ (Algorithm 2), we transform the stochastic process (43) to be deterministic as $\ln \left(\beta_{t+1}\right)=\rho \ln \left(\beta^{*}\right)+(1-\rho) \ln \left(\beta_{t}\right)$, and then transform the system of equilibrium equations (44) and (46)-(52) to be deterministic by canceling their corresponding expectation operator, and choose $T=200$ in the transformed system (5).

In the approximation step of Algorithm 2, since the control variables $\left(c_{t}, \ell_{t}, r_{t}, q_{t}, z_{t}, \pi_{t}\right)$ can be simply substituted, we only need to approximate three control variables $\left(\chi_{t, 1}, \chi_{t, 2}, y_{t}\right)$ over two state variables $\left(v_{t}, \beta_{t}\right)$. We use the relative $\mathcal{L}^{1}$ norm (relative to the steady state values given in Appendix $\mathrm{C},\left(v^{*}, \chi_{1}^{*}, \chi_{2}^{*}, y^{*}\right)$, respectively) in the objective of (5). We use the tensor grid 
Table 10: Errors of the NLCEQ solution with degree- $D$ complete Chebyshev polynomials for the New Keynesian DSGE model with ZLB

\begin{tabular}{c|cccc}
\hline & $D=4$ & $D=6$ & $D=8$ & $D=10$ \\
\hline $\mathcal{L}^{\infty}$ Global Error & $4.4(-3)$ & $3.1(-3)$ & $2.2(-3)$ & $1.8(-3)$ \\
$\mathcal{L}^{1}$ Global Error & $8.1(-4)$ & $6.0(-4)$ & $5.6(-4)$ & $4.6(-4)$ \\
\hline
\end{tabular}

Note: $\zeta(-j)$ means $\zeta \times 10^{-j}$.

of Chebyshev nodes ( $D+1$ nodes in each dimension) and degree- $D$ complete Chebyshev polynomials to approximate the policy functions. We want to get a solution over the state space $[1,1.04] \times[0.96,1.03]$, a slightly wider domain than the one used in Guerrieri and Iacoviello (2015), so it will have a higher chance of a binding ZLB. Thus we choose the approximation domain of $(v, \beta)$ as $[1,1.045] \times[0.936,1.056]$ so that next simulated states transited from current states in $[1,1.04] \times[0.96,1.03]$ using (49) and (43) will be inside the approximation domain.

Table 10 reports unit-free errors of the NLCEQ solution for various degrees $D$. The errors are computed on the domain $[1,1.04] \times[0.96,1.03]$. The global errors are defined in a similar way in Subsection 3.1, while we need to estimate the unit-free errors for the equations (44), (46), and (47), where we use the 15-point Gauss-Hermite quadrature rule to estimate the integrations. We see that they achieve $O\left(10^{-3}\right)$ errors in $\mathcal{L}^{\infty}$ or $O\left(10^{-4}\right)$ errors in $\mathcal{L}^{1}$, and a higher degree approximation improves the accuracy.

We also solve the model with the order-1 (linearization) and order- 2 perturbation methods. The order-1 perturbation gives an error 0.011 in $\mathcal{L}^{\infty}$ and 0.0014 in $\mathcal{L}^{1}$. The order- 2 perturbation does not improve the accuracy, its error is 0.012 in $\mathcal{L}^{\infty}$ and 0.0012 in $\mathcal{L}^{1}$. NLCEQ is almost one digit more accurate than the perturbation methods.

The comparison between NLCEQ and the linearization method is also shown in Figure 4, which shows the global errors of NLCEQ with degree-10 complete Chebyshev polynomials and of the order-1 perturbation method when $\beta=0.96,0.994,1.03$. We see that NLCEQ is always more accurate 
Figure 4: Errors of the NLCEQ solution for the New Keynesian DSGE model with ZLB

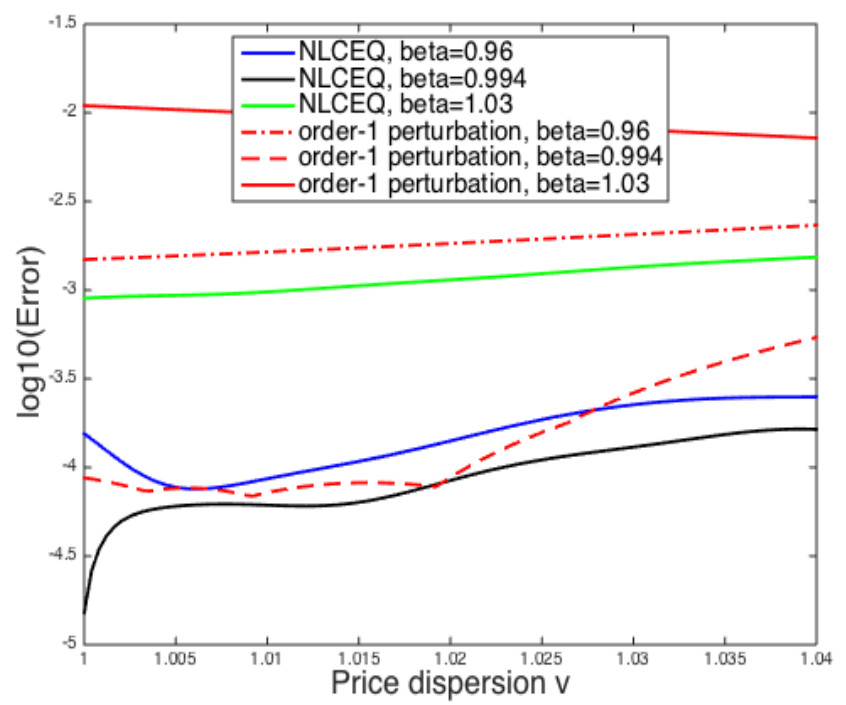

than the linearization method. The errors are smaller when $\beta$ is closer to steady $\beta^{*}=0.994$. When $\beta$ is the largest (i.e., $\beta=1.03$ ), the errors are the largest because a higher $\beta$ implies a higher chance of a binding ZLB (about $24 \%$ state points in $[1,1.04] \times[0.96,1.03]$ have a binding ZLB).

Figure 5 shows impulse responses of interest rate $r_{t}$, inflation $\pi_{t}$ (the figure shows the net inflation rate in percent, i.e., $\left.100\left(\pi_{t}-1\right) \%\right)$, and output $y_{t}$ (the figure shows deviation of output from the steady state in percent, i.e., $100\left(y_{t} / y_{s s}-1\right) \%$ ) to a shock of discount factor $\beta_{1}$ (with $v_{1}=v_{s s}$ ). The left panel of the figure shows responses to a shock that brings $\beta_{1}$ up to 1.03, and the right panel shows responses to a shock that brings $\beta_{1}$ down to 0.96 . We see that the interest rate hits the ZLB in the first four periods in the left panel, and all three responses $\left(r_{t}, \pi_{t}, y_{t}\right)$ are decreasing functions of $\beta$ ( $\beta_{t}$ decreases along time $t$ in the left panel, and $\beta_{t}$ increases along time $t$ in the right panel), and they are almost steady after 20 periods (i.e., 5 years).

We also show that NLCEQ (Algorithm 2) can solve deterministic com- 
Figure 5: Impulse responses to a shock of discount factor
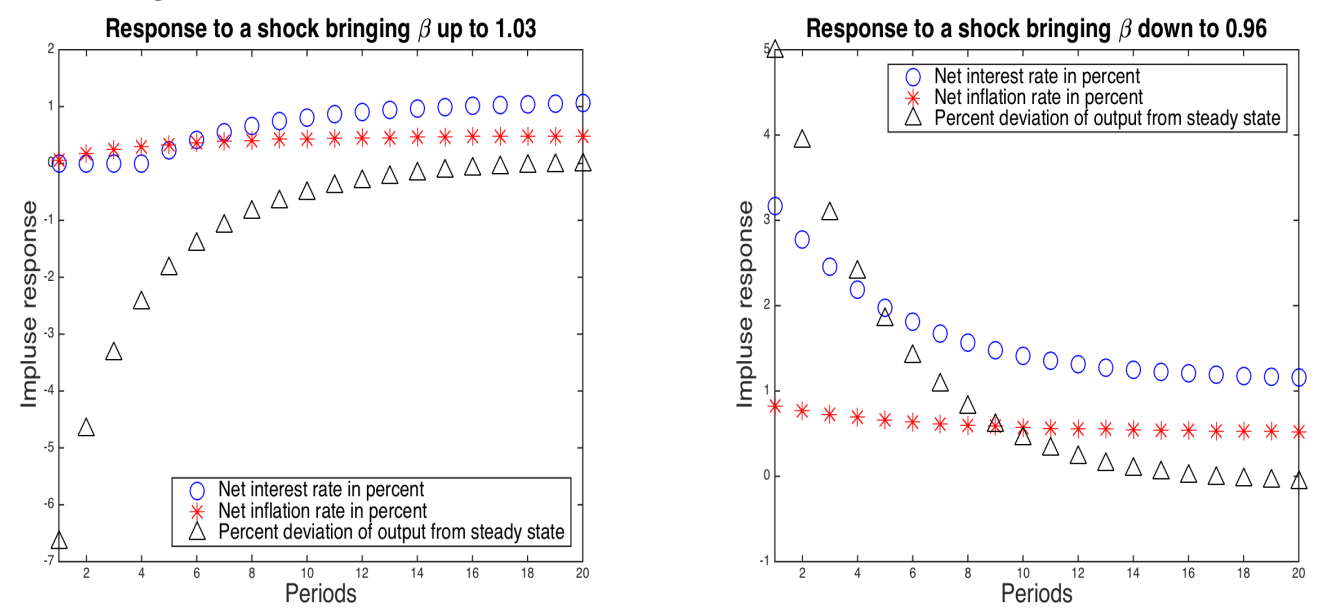

Table 11: Errors of the NLCEQ solution with degree- $D$ complete Chebyshev polynomials for the New Keynesian DSGE model with ZLB and $\sigma=0$

\begin{tabular}{c|cccc}
\hline & $D=10$ & $D=20$ & $D=50$ & $D=100$ \\
\hline $\mathcal{L}^{\infty}$ Global Error & $1.3(-3)$ & $7.3(-4)$ & $5.2(-4)$ & $1.7(-4)$ \\
$\mathcal{L}^{1}$ Global Error & $2.3(-4)$ & $8.5(-5)$ & $4.6(-5)$ & $1.3(-5)$ \\
\hline
\end{tabular}

Note: $\zeta(-j)$ means $\zeta \times 10^{-j}$.

petitive equilibrium problems very accurately. Table 11 lists global errors in $\mathcal{L}^{\infty}$ norm over $[1,1.04] \times[0.96,1.03]$ for the New Keynesian DSGE problem with ZLB and $\sigma=0$. We choose a large $T=300$ and a higher degree approximation in order to get a higher accuracy. We see that NLCEQ reaches about 4-digit accuracy in $\mathcal{L}^{\infty}$ for the optimal policy functions to the deterministic competitive equilibrium problem.

\section{Conclusion}

We have shown that NLCEQ can be applied to solve dynamic stochastic problems with acceptable accuracy when we combine modern approximation optimization methods with parallel computing architectures. Examples 
include high-dimensional optimal stochastic growth problems up to four hundred state variables and three problems with occasionally binding constraints, including a dynamic stochastic model of food and clean energy, and a New Keynesian DSGE model with zero lower bound. This approach greatly expands the range of problems that can be solved well globally, and clearly dominates any form of linearization. 


\section{References}

[1] Adjemian, S., Bastani, H., Karamé, F., Juillard, M., Maih, J., Mihoubi, F., Perendia, G., Ratto, M., Villemot, S. (2011). Dynare: reference manual version 4. Dynare Working Papers 1, CEPREMAP.

[2] Aruoba, S.B., J. Fernandez-Villaverde, and J.F. Rubio-Ramirez (2006). Comparing solution methods for dynamic equilibrium economies. Journal of Economic Dynamics and Control, 30, 2477-2508.

[3] Andreoni, J., and A. Levinson (2001). The simple analytics of the environmental Kuznets curve. Journal of Public Economics 80, 269-286.

[4] Bellman, R. (1957). Dynamic Programming. Princeton University Press.

[5] Benigno, P., and M. Woodford (2012). Linear-quadratic approximation of optimal policy problems. Journal of Economic Theory 147(1), 1-42.

[6] Bertsekas, D. (2005). Dynamic Programming and Optimal Control, Vols. I. Athena Scientific.

[7] Bertsekas, D. (2007). Dynamic Programming and Optimal Control, Vols. II. Athena Scientific.

[8] Brumm, J., and S. Scheidegger (2014). Using adaptive sparse grids to solve high-dimensional dynamic models. SSRN working paper 2349281.

[9] Byrd, R.H., J. Nocedal, R.A. Waltz (2006). KNITRO: An Integrated Package for Nonlinear Optimization. http://www.ziena.com/papers/ integratedpackage.pdf. Technical report, Ziena.

[10] Cai, Y., and K.L. Judd (2012). Dynamic programming with shapepreserving rational spline Hermite interpolation. Economics Letters, 117(1), 161-164. 
[11] Cai, Y., and K.L. Judd (2013). Shape-preserving dynamic programming. Mathematical Methods of Operations Research, 77(3), 407-421.

[12] Cai, Y. and K.L. Judd (2014). Advances in numerical dynamic programming and new applications. Chapter 8 in: Handbook of Computational Economics, Vol. 3, ed. by K. Schmedders and K.L. Judd, Elsevier.

[13] Cai, Y., and K.L. Judd (2015). Dynamic programming with Hermite approximation. Mathematical Methods of Operations Research, 81, 245267.

[14] Cai, Y., K.L. Judd, and T.S. Lontzek (2015a). The social cost of carbon with economic and climate risks. Working paper, arXiv preprint arXiv:1504.06909.

[15] Cai, Y., K.L. Judd, G. Thain, and S. Wright (2015b). Solving dynamic programming problems on computational grid. Computational Economics, 45(2), 261-284..

[16] Cai, Y., K.L. Judd and R. Xu (2013). Numerical solutions of dynamic portfolio optimization with transaction costs. NBER working paper 18709.

[17] Cai, Y., J. Steinbuks, J. Elliott, and T.W. Hertel (2014). The effect of climate and technological uncertainty in crop yields on the optimal path of global land use. Policy Research Working Paper Series 7009, The World Bank.

[18] Caldara, D., J. Fernández-Villaverde, J.F. Rubio-Ramírez, and W. Yao (2012). Computing DSGE models with recursive preferences and stochastic volatility. Review of Economic Dynamics, 15, 188-206.

[19] Chakravorty, U., B. Magne and M. Moreaux (2008). A dynamic model of food and clean energy. Journal of Economic Dynamics and Control, $32(4), 1181-1203$. 
[20] Chakravorty, U., Hubert, M.-H., and Nostbakken, L. (2012). The long run impact of biofuels on food prices. Working Paper 2012-11, University of Alberta, Department of Economics.

[21] Christiano, L.J., and J.D.M. Fisher (2000). Algorithms for solving dynamic models with occasionally binding constraints. Journal of Economic Dynamics and Control, 24, 1179-1232.

[22] Czyzyk, J., M.P. Mesnier, and J.J. Moré (1998). The NEOS server. IEEE Computational Science \& Engineering, 5: 68-75.

[23] Del Negro, M., F. Schorfheide, F. Smets, and R. Wouters (2007). On the fit of new Keynesian models. Journal of Business 83 Economic Statistics, 25 (2), 123-143.

[24] Den Haan, W.J., K.L. Judd and M. Juillard (2011). Computational suite of models with heterogeneous agents II: Multi-country real business cycle models. Journal of Economic Dynamics and Control, 35, 175-177.

[25] Den Haan, W.J., and J. De Wind (2012). Nonlinear and stable perturbation-based approximations. Journal of Economic Dynamics and Control 36(10), 1477-1497.

[26] Drud, A.S. (1996). CONOPT: A System for Large Scale Nonlinear Optimization. ARKI Consulting and Development A/S, Bagsvaerd, Denmark.

[27] Epstein, L.G., and S.E. Zin (1989). Substitution, risk aversion, and the temporal behavior of consumption and asset returns: a theoretical framework. Econometrica, 57(4), 937-969.

[28] Fernández-Villaverde, J., and J.F. Rubio-Ramírez (2006). Solving DSGE models with perturbation methods and a change of variables. Journal of Economic Dynamics and Control 30(12), 2509-2531. 
[29] Fernández-Villaverde, J., G. Gordon, P. Guerrón-Quintana, and J.F. Rubio-Ramírez (2015). Nonlinear adventures at the zero lower bound. Journal of Economic Dynamics and Control 57, 182-204.

[30] Fourer, R., D. M. Gay, B. W. Kernighan (2003). AMPL: A Modeling Language for Mathematical Programming, 2nd ed. Duxbury Press, Pacific Grove, CA.

[31] Gali, J. (2008). Monetary Policy, Inflation, and the Business Cycle: An Introduction to the New Keynesian Framework. Princeton University Press, Princeton, NJ.

[32] Gaspar, J., and K.L. Judd (1997). Solving large-scale rationalexpectations models. Macroeconomic Dynamics, 1(1), 45-75.

[33] Gill, P., W. Murray, and M.A. Saunders (2005). SNOPT: An SQP algorithm for largescale constrained optimization. SIAM Review, 47(1), 99-131.

[34] Gropp, W., and J.J. Moré (1997). Optimization environments and the NEOS server. M. D. Buhmann, A. Iserles, eds. Approximation Theory and Optimization: Tributes to M. J. D. Powell. Cambridge University Press, Cambridge, UK, 167-182.

[35] Guerrieri, L., and M. Iacoviello (2015). OccBin: A toolkit for solving dynamic models with occasionally binding constraints easily. Journal of Monetary Economics, 70, 22-38.

[36] Hansen, L.P., and T.J. Sargent (2005). Certainty equivalence and model uncertainty. Proceedings of the March 2004 Conference "Models 83 Monetary Policy: Research in the Tradition of Dale Henderson, Richard Porter, and Peter Tinsley", Board of Governors of the Federal Reserve System, 17-38. 
[37] Infanger, G. (2006). Dynamic asset allocation using a stochastic dynamic programming approach. In Handbook of Asset and Liability Management, volume 1, North Holland.

[38] Jin, H.-H., and K.L. Judd (2002). Perturbation methods for general dynamic stochastic models. Working paper, Stanford University.

[39] Judd, K.L. (1998). Numerical Methods in Economics. The MIT Press.

[40] Juillard, M., and S. Villemot (2011). Multi-country real business cycle models: Accuracy tests and test bench. Journal of Economic Dynamics and Control, 35, 178-185.

[41] Kim, J., S. Kim, E. Schaumburg, and C.A. Sims (2008). Calculating and using second-order accurate solutions of discrete time dynamic equilibrium models. Journal of Economic Dynamics and Control 32(11), 33973414.

[42] Kollmann, R., S. Maliar, B.A. Malin, and P. Pichler (2011). Comparison of solutions to the multi-country real business cycle model. Journal of Economic Dynamics and Control, 35, 186-202.

[43] Magill, J.P.M. (1977). A local analysis of N-sector capital accumulation under uncertainty. Journal of Economic Theory, 15: 211-219.

[44] Malin, B.A., Krueger, D., and Kubler, F. (2011). Solving the multicountry real business cycle model using a Smolyak collocation method. Journal of Economic Dynamics and Control 35, 229-239.

[45] Maliar, L., and S. Maliar (2015). Merging simulation and projection approaches to solve high-dimensional problems with an application to a new Keynesian model. Quantitative Economics, 6, 1-47. 
[46] McCarl, B., et al. (2011). McCarl Expanded GAMS user guide version 23.6. http://www.gams.com/mccarl/mccarlhtml/. Accessed 18 January 2015 .

[47] Miranda, M.J., and P.L. Fackler (2002). Applied computational economics and finance. MIT press.

[48] Nordhaus, W.D., and J. Boyer. (2003). Warming the world: economic models of global warming. MIT press.

[49] Rust, J. (2008). Dynamic Programming. In: New Palgrave Dictionary of Economics, ed. by Steven N. Durlauf and Lawrence E. Blume. Palgrave Macmillan, second edition.

[50] Schmitt-Grohe, S., and M. Uribe (2004). Solving dynamic general equilibrium models using a second-order approximation to the policy function. Journal of Economic Dynamics and Control 28(4), 755-775.

[51] Simon, H. A. (1956). Dynamic programming under uncertainty with a quadratic criterion function. Econometrica, 24(1), 74-81.

[52] Smets, F., and R. Wouters (2007). Shocks and frictions in US business cycles: A Bayesian DSGE approach. American Economic Review, 97(3), 586-606.

[53] Smolyak, S. (1963). Quadrature and interpolation formulas for tensor products of certain classes of functions. Soviet Mathematics, Doklady 4, 240-243.

[54] Steinbuks, J., and Hertel, T.W. (2014). Confronting the food-energyenvironment trilemma: global land use in the long run. Environmental and Resource Economics, in press, doi:10.1007/s10640-014-9848-y.

[55] Theil, H. (1957). A note on certainty equivalence in dynamic planning. Econometrica, 25(2), 346-349. 
[56] Tauchen, G. (1986). Finite state Markov-chain approximations to univariate and vector autoregressions. Economic Letters, 20, 177-181.

[57] Taylor, J. (1993). Discretion versus policy rules in practice. CarnegieRochester Conference Series on Public Policy, 39, 195-214.

[58] Woodford, M. (2003). Interest and Prices: Foundations of a Theory of Monetary Policy. Princeton University Press.

[59] Xepapadeas, A. (2005). Economic growth and the environment. Chapter 23 in Handbook of Environmental Economics, vol. 3, 1219-1271, ed. by K.-G. Mäler and J.R. Vincent, Elsevier. 


\section{Appendix A-Approximation}

An approximation scheme approximates a function $F(\mathbf{x})$ with $\hat{F}(\mathbf{x} ; \mathbf{b})=$ $\sum_{j=0}^{n} b_{j} \phi_{j}(\mathbf{x})$ for some vector of parameters $\mathbf{b}$. A spectral method uses globally nonzero basis functions $\phi_{j}(\mathbf{x})$. Examples of spectral methods include ordinary or Chebyshev polynomial approximation. In contrast, a finite element method uses local basis functions where for each $j$ the basis function $\phi_{j}(\mathbf{x})$ is zero except on a small part of the approximation domain. Examples of finite element methods include piecewise linear interpolation, cubic splines, and B-splines. See Cai and Judd (2014, 2015) and Judd (1998) for more details.

\section{Chebyshev Polynomial Approximation}

Chebyshev polynomials on $[-1,1]$ are defined as $\phi_{j}(z)=\cos \left(j \cos ^{-1}(z)\right)$. The Chebyshev polynomials on a general interval $\left[x_{\min }, x_{\max }\right]$ are defined as $\phi_{j}\left(\left(2 x-x_{\min }-x_{\max }\right) /\left(x_{\max }-x_{\min }\right)\right)$ for $j \geq 0$, and are orthogonal under the weighted inner product $\langle f, g\rangle=\int_{x_{\min }}^{x_{\max }} f(x) g(x) w(x) d x$ with the weighting function

$$
w(x)=\left(1-\left(\frac{2 x-x_{\min }-x_{\max }}{x_{\max }-x_{\min }}\right)^{2}\right)^{-1 / 2} .
$$

A degree $D$ Chebyshev polynomial approximation for $V(x)$ on $\left[x_{\min }, x_{\max }\right]$ is

$$
\hat{V}(x ; \mathbf{b})=\sum_{j=0}^{D} b_{j} \phi_{j}\left(\frac{2 x-x_{\min }-x_{\max }}{x_{\max }-x_{\min }}\right),
$$

where $b_{j}$ are the Chebyshev coefficients.

The canonical Chebyshev nodes on $[-1,1]$ are $z_{i}=-\cos ((2 i-1) \pi /(2 m))$ for $i=1, \ldots, m$, and the corresponding Chebyshev nodes adapted for the general interval $\left[x_{\min }, x_{\max }\right]$ are $x_{i}=\left(z_{i}+1\right)\left(x_{\max }-x_{\min }\right) / 2+x_{\min }$. If we have Lagrange data $\left\{\left(x_{i}, v_{i}\right): i=1, \ldots, m\right\}$ with $v_{i}=V\left(x_{i}\right)$, then the coefficients 
$b_{j}$ in (53) are

$$
b_{j}=\frac{2}{m} \sum_{i=1}^{m} v_{i} \phi_{j}\left(z_{i}\right), \quad j=1, \ldots, D,
$$

and $b_{0}=\sum_{i=1}^{m} v_{i} / m$. The method is called the Chebyshev regression algorithm in Judd (1998).

\section{Multidimensional Complete Chebyshev Approximation}

In a $d$-dimensional approximation problem, the domain of the approximation function will be

$$
\left\{\mathbf{x}=\left(x_{1}, \ldots, x_{d}\right): x_{\min , i} \leq x_{i} \leq x_{\max , i}, i=1, \ldots d\right\},
$$

Let $\mathbf{x}_{\min }=\left(x_{\min , 1}, \ldots, x_{\min , d}\right)$ and $\mathbf{x}_{\max }=\left(x_{\max , 1}, \ldots, x_{\max , d}\right)$. We let $\left[\mathbf{x}_{\min }, \mathbf{x}_{\max }\right]$ denote the domain. Let $\alpha=\left(\alpha_{1}, \ldots, \alpha_{d}\right)$ be a vector of nonnegative integers. Let $\phi_{\alpha}(\mathbf{z})$ denote the product $\prod_{i=1}^{d} \phi_{\alpha_{i}}\left(z_{i}\right)$ for $\mathbf{z}=\left(z_{1}, \ldots, z_{d}\right) \in[-1,1]^{d}$. Let

$$
Z(\mathbf{x})=\left(\frac{2 x_{1}-x_{\min , 1}-x_{\max , 1}}{x_{\max , 1}-x_{\min , 1}}, \ldots, \frac{2 x_{d}-x_{\min , d}-x_{\max , d}}{x_{\max , d}-x_{\min , d}}\right)
$$

for any $\mathbf{x}=\left(x_{1}, \ldots, x_{d}\right) \in\left[\mathbf{x}_{\min }, \mathbf{x}_{\max }\right]$. With this notation, the degree- $D$ complete Chebyshev approximation for $V(\mathbf{x})$ is

$$
\hat{V}(\mathbf{x} ; \mathbf{b})=\sum_{\alpha \geq 0,|\alpha| \leq D} b_{\alpha} \phi_{\alpha}(Z(\mathbf{x}))
$$

where $|\alpha|=\sum_{i=1}^{D} \alpha_{i}$. This is a degree $D$ polynomial, and has $\left(\begin{array}{c}d+D \\ D\end{array}\right)$ terms. 


\section{Appendix B-Equilibrium Conditions in the New Keynesian DSGE Model}

The final-good firm buys intermediate goods $y_{i, t}$ from intermediate firms to produce a final good $y_{t}$ with the following production function

$$
y_{t}=\left(\int_{0}^{1} y_{i, t}^{\frac{\alpha-1}{\alpha}} d i\right)^{\frac{\alpha}{\alpha-1}}
$$

then sell $y_{t}$ at a price $p_{t}$. Let $p_{i, t}$ be prices of $y_{i, t}$, then the final-good firm chooses $y_{i, t}$ to maximize its profit:

$$
\max _{y_{i, t}} p_{t} y_{t}-\int_{0}^{1} p_{i, t} y_{i, t} d i .
$$

Its first-order condition implies

$$
y_{i, t}=y_{t}\left(\frac{p_{i, t}}{p_{t}}\right)^{-\alpha} .
$$

The intermediate firms rent labor supply $\ell_{i, t}$ from the household with a wage rate $w_{t}$ and produce $y_{i, t}$ with a simple production function

$$
y_{i, t}=\ell_{i, t}
$$

and sell $y_{i, t}$ at a price $p_{i, t}$ to the final-good firm. The intermediate firms are assumed to have Calvo-type prices: a fraction $1-\theta$ of the firms have optimal prices and the remaining fraction $\theta$ of the firms keep the same price as in the previous period.

A re-optimizing intermediate firm $i \in[0,1]$ chooses its price $p_{i, t}$ to maximize the current value of profit over the time when the optimal $p_{i, t}$ remains 
effective:

$$
\max _{p_{i, t}} \mathbb{E}_{t}\left\{\sum_{j=0}^{\infty}\left(\prod_{k=0}^{j} \beta_{t+k}\right) \lambda_{t+j} \theta^{j}\left(p_{i, t} y_{i, t+j}-w_{t+j} \ell_{i, t+j}\right)\right\}
$$

subject to the constraints $y_{i, t+j}=\ell_{i, t+j}$ from (57) and

$$
y_{i, t+j}=y_{t+j}\left(\frac{p_{i, t}}{p_{t+j}}\right)^{-\alpha}
$$

from (56) by letting $p_{i, t+j}=p_{i, t}$. Here $\lambda_{t}$ is the Lagrange multiplier of the budget constraint (41). From the first-oder conditions of the household problem (42), $\lambda_{t}$ satisfies the following equation:

$$
\lambda_{t}=\frac{1}{p_{t} c_{t}}
$$

The first-order condition of the re-optimizing intermediate firm problem (58) implies

$$
\mathbb{E}_{t}\left\{\sum_{j=0}^{\infty}\left(\prod_{k=0}^{j} \beta_{t+k}\right) \lambda_{t+j} \theta^{j} p_{t+j}^{\alpha} y_{t+j}\left(p_{i, t}-\frac{\alpha}{\alpha-1} w_{t+j}\right)\right\}=0
$$

Let $\pi_{t, j}=p_{t+j} / p_{t}$. From (45), (59) and (60), for any re-optimizing firm $i$ we have

$$
\frac{p_{i, t}}{p_{t}} \equiv q_{t}=\frac{\alpha \chi_{t, 1}}{(\alpha-1) \chi_{t, 2}}
$$

where

$$
\begin{aligned}
\chi_{t, 1} & \equiv y_{t} \ell_{t}^{\eta}+\mathbb{E}_{t}\left\{\sum_{j=1}^{\infty}\left(\prod_{k=1}^{j} \beta_{t+k}\right) \theta^{j} \pi_{t, j}^{\alpha} y_{t+j} \ell_{t+j}^{\eta}\right\} \\
\chi_{t, 2} & \equiv \frac{y_{t}}{c_{t}}+\mathbb{E}_{t}\left\{\sum_{j=1}^{\infty}\left(\prod_{k=1}^{j} \beta_{t+k}\right) \theta^{j} \pi_{t, j}^{\alpha-1} \frac{y_{t+j}}{c_{t+j}}\right\}
\end{aligned}
$$


We have the recursive formulas for $\chi_{t, 1}$ and $\chi_{t, 2}$ :

$$
\begin{aligned}
\chi_{t, 1} & =y_{t} \ell_{t}^{\eta}+\theta \mathbb{E}_{t}\left\{\beta_{t+1} \pi_{t+1}^{\alpha} \chi_{t+1,1}\right\} \\
\chi_{t, 2} & =\frac{y_{t}}{c_{t}}+\theta \mathbb{E}_{t}\left\{\beta_{t+1} \pi_{t+1}^{\alpha-1} \chi_{t+1,2}\right\}
\end{aligned}
$$

From (55) and (56), we have

$$
\begin{aligned}
p_{t} & =\left(\int_{0}^{1} p_{i, t}^{1-\alpha} d i\right)^{\frac{1}{1-\alpha}} \\
& =\left((1-\theta)\left(q_{t} p_{t}\right)^{1-\alpha}+\theta \int_{0}^{1} p_{i, t-1}^{1-\alpha} d i\right)^{\frac{1}{1-\alpha}} \\
& =\left((1-\theta)\left(q_{t} p_{t}\right)^{1-\alpha}+\theta p_{t-1}^{1-\alpha}\right)^{\frac{1}{1-\alpha}}
\end{aligned}
$$

as

$$
p_{t-1}=\left(\int_{0}^{1} p_{i, t-1}^{1-\alpha} d i\right)^{\frac{1}{1-\alpha}}
$$

This follows that

$$
q_{t}=\left(\frac{1-\theta \pi_{t}^{\alpha-1}}{1-\theta}\right)^{\frac{1}{1-\alpha}}
$$

From (56), (57) and the following market clearing condition

$$
\ell_{t}=\int_{0}^{1} \ell_{i, t} d i
$$


we get

$$
\begin{aligned}
v_{t+1} \equiv \ell_{t} / y_{t} & =\int_{0}^{1}\left(\frac{p_{i, t}}{p_{t}}\right)^{-\alpha} d i \\
& =(1-\theta) q_{t}^{-\alpha}+\theta \int_{0}^{1}\left(\frac{p_{i, t-1}}{p_{t}}\right)^{-\alpha} d i \\
& =(1-\theta) q_{t}^{-\alpha}+\theta \pi_{t}^{\alpha} \int_{0}^{1}\left(\frac{p_{i, t-1}}{p_{t-1}}\right)^{-\alpha} d i \\
& =(1-\theta) q_{t}^{-\alpha}+\theta \pi_{t}^{\alpha} v_{t}
\end{aligned}
$$

\section{Appendix C-Steady State of the New Key- nesian DSGE Model}

From (63), the steady state of $\chi_{t, 2}$ is

$$
\chi_{2}^{*}=\frac{1}{\left(1-s_{g}\right)\left(1-\theta \beta^{*}\left(\pi^{*}\right)^{\alpha-1}\right)}
$$

with the given $\pi^{*}=1.005$. From (61) and (64), the steady state of $\chi_{t, 1}$ is

$$
\chi_{1}^{*}=\chi_{2}^{*} q^{*} \frac{\alpha-1}{\alpha}
$$

with

$$
q^{*}=\left(\frac{1-\theta\left(\pi^{*}\right)^{\alpha-1}}{1-\theta}\right)^{\frac{1}{1-\alpha}}
$$

and from (65) the steady state of $v_{t}$ is

$$
v^{*}=\frac{(1-\theta)\left(q^{*}\right)^{-\alpha}}{1-\theta\left(\pi^{*}\right)^{\alpha}}
$$


Therefore, from $v_{t}=\ell_{t} / y_{t}$ and (62), we get

$$
y^{*}=\left(\frac{\chi_{1}^{*}\left(1-\theta \beta^{*}\left(\pi^{*}\right)^{\alpha}\right)}{\left(v^{*}\right)^{\eta}}\right)^{\frac{1}{1+\eta}}
$$

